\title{
Compte rendu des travaux du Comité Technique de la Société Hydrotechnique de France
}

\author{
Meetings of the technical committee
}

\section{Session des 18 et 19 mars 1953}

Le Comité Technique a siégé les 18 et 19 mars 1953 , 12, place des Etats-Unis, Paris $\left(16^{\mathrm{e}}\right)$.

Ont participé aux travaux de cette session:
a) Conseil d'administration :

MM. Amleret, Delattre, Garier, Bouchayer, Chevrier, Devun, Hupner, Nizeny, Legros représentant M. Coyne.

\section{b) Adhérents :}

Electricité de France, Compagnie Nationale du Rhône, Etablissements Neyrpic, Compagnie de Fives-Lille. Entreprises Métropolitaines et Coloniales, Entreprises des Grands Travaux Hydrauliques, Société Lyonnaise des Eaux et de l'Eclairage, Entreprise Bachy, Bureau d'Etudes Coyne et J. Bellier, Direction des Travaux Publies de Tunisie.

c) Comité Technique :

MM. Barrillon, Bergeron, Radiguer, Serra, Rúm:NIERTAS, AILARD, BENOIST, BOURGUIGNON, BOUVARD, Braudeat, Chabert, Crescent, Duffayt, Dupouy,
Esclangon, Ferrandon, Ferry, Fontalne, Frolow, Frontard, Germain, Gibert, Gougenheim, Henin, JUPILLAT, KaMPE DE FÉntet, LaBaye, Laftéche, LaNglois, Lescail, Lomband, Mattre, Méo, Michon, Morlat, Pandé, Panodi, Suquet, Varlet, Vennin, VIBERT, WAHL.

MM. Devimeux et Mondiliat représentant respectivement MM. J. LAUNENT et Koch.
d) Invités :

MHe Garenc, MM. Frinink et Faner (Hollande), MM. Gemaehling, Ch. Beav, Rovldeay, Roment, Wubrate, Melot, Huard de ta Manny, Dreyrus, Cahen, AIT OUyahia, Bafoun.

\section{S'étaient excusés :}

MM. Massé, Archambault, Barnithe, Brosset, La Bovnhis, Brand, Callueux, Covtaline, Covteaun, Coyne, Drounin, Escande, Gullo, Grouet, Gonmon Foster, Luxo, Cl. Marcello, Ménier, Mressines du Sourbien, Montagne, Oudin, Ronien, de Rovvhlis, Schlak, Thisse, Tison, Vantroys.

\section{SÉANCE DU MERCREDI MATIN 18 MARS 1953}

La séance est ouverte à 9 h. 15 sous la présidence de M. Barrillon.

En communiquant les exeuses des Membres ou invités précités, M. le Président présente à M. Coutrane, souffrant, les voux que le Comité technique forme pour son rétablissement, salue la présence de MM. FrIJLINk et FABER, Ingénieurs au Waterloopluundig Laboratorium de Delft (Hollande), représentant $M$. Thussse, occupé à la reconstruction des digues des Pays-Bas, et espère que ces travaux donneront lieu à une communication du Waterloopkundig Laboratorium.

\section{VIE.DE LA SOCIÉTÉ}

M. le président donne ensuite connaissance des faits ci-après, relatifs à la vie de la Societé :

\section{Adoption du procès-verbal de la session des 25,26 et 27 novembre 1952}

Ce process-verbal, mis en lecture pendant toute la durée de la présente session n'a donné lieu à aucune observation. Il est donc adopté ipso facto. 


\section{Promotions et nomination}

Sont promus Commandeurs de la Légion d'honneur : M. Gaspard, Directeur Général de l'Electricité de France. M. Defatrne, Directeur Général de la Compagnie Nationale du Rhône.

Sont nommés Chevaliers de la Légion d'honneur:

M. NuERx, Divecteur Adjoint des Etudes et Recherches à E.D.F.

M. Fennandien, Secrétaire Général de la S.H.F.

Est nommé Inspecteur Général des Ponts et Chaussées :

M. Gurnt, ancien Ingénieur en Chef du Service Maritime de la Loire-Inféricure.

M. le Président présente aux nouveaux promus les félicitations du Comité Technique. (Applaudissements.)

\section{Admissions au Comité Technique}

M. le Président présente les personnalités ci-après en vue de leur admission au Comité Technique :

M. Couteaud, Ingénieur en Ghef des Ponts et Chaussées, Directeur du Port de Marseille.

M. RoBert, Ingẻnieur en Chef des Ponts et Chaussees, Chef de la $7^{\mathrm{e}}$ Circonscription Electrique, Dijon.

M. Sreqfaled, Ingénieur en Chef des Ponts et Chaussées, Chef du Service Maritime de la Loire-Inférieure.

M. Vantroys, Ingénieur Hydrographe en Chef du Cadre de Réserve, Ingénieur en Chef à E.D.F., Chef du Service d'Etudes pour l'Utilisation des Marées.

M. Roulleav, Ingénieur en Chef de la Météorologie, Chef de l'Etahlissement d'Etudes et de Recherches Météorologiques.

M. Arlenx, Ingénieux en Chef de la Météorologie Nationale.

M. Ballade, Ingénieur des Ponts et Chaussées, Service Maritime de la Loire-Inférieure.

Mhe Garenc, Attaché de Recherches au C.N.R.S.

Ces admissions approuvées par le Comité Technique seront soumises à la ratification du Conseil d'Administration.

\section{Documents reçus depuis la dernière sesnion}

M. le Président donne la liste des documents reçus depuis la dernière session, que nos Membres peuvent consulter au Secrétariat de Ja S.H.F.

\section{Prix Henri Milon (Monographie hydrologique)}

Le 12 décembre 1952, les Membres du Jury du Prix Henri Milon ont invité les auteurs d'ouvrages primés les années précédentes à une réunion commune dont les conclusions seront commmiquées aux candidats dans la circulaire annuelle relative à ce Prix.

\section{Commission des pertes de charge}

La Commission pour l'étude des pertes de charge s'est réunie le 19 décembre 1952.

La documentation sur cette question a été mise à jour notamment par MM. Roussecer et Pravaz.

M. Réménéras a fait part d'observations faites. en Laboratoire et au cour's d'essais d'usines hydroélectriques, notamment sur les pertes de charge dues à des dépôts bỉologiques, et sur. Ia prédétermination par circulation d'air des pertes de charge dans les galeries en charge d'aménagements hydrauliques.

M. Mathieu a rendu compte du comportement des enduits bitumineux avec le temps.

M. Renovard a présenté un abaque de pertes de charge courantes pour le propane et le gaz de ville, et N. Chapus, un catalogue des pertes de charge singulieres pour les fluides gazeux.
La Commission a adopté les conclusions suivantes :

$1^{\circ}$ Afin d'étudier leurs variations avec le temps, il serail désirable que les pertes de charge soient mesurées d'une façon continue. Faute de mieux, une ou plusieurs usines pourraient suivre de très près pendant une année au moins, les fluctuations de in perte de charge;

$2^{\circ}$ Il est recommandé anx auteurs publiant des résultats de signaler tous les détails de mesures et observations et, notamment, les résultats de l'examen minéralogique et biologique des dépôts sur les parois;

$3^{\circ}$ Utilité de traduire les abaques de pertes de charge en tableaux ou barèmes;

4 "Concentrer les efforts sur l'établissement d'un nouveau tableau de pertes de charge singulières.

M. le Président signale, en outre, à propos des pertes de charge :

a) Un ouvrage de Schuching sur la couche limite;

b) Les essais sur la vérification des lois de frottement dans les bateaux propulsés par réacteurs externes pour éviter l'intéraction entre l'hélice et la carène : essais sur modèles réduits à plusieurs échelles différentes et sur navire réel exécutés en Angleterre (Naval Architectes), en Hollande ef aux Etats-Unis.

\section{Section d'hydraulique fluviale et maritime}

La Section s'est réunie le 15 janvier 1953 sous la présidence de M. de Rouville.

M. Gibrat a exposé les nouveaux aspects de la question de l'utilisation des marées (énergie naturclle des marées, énergie dissipée par les marées, énergie accumulée) en s'attachant à montrer l'importance de l'amplitude et de la résonance ainsi que l'influence de l'exploitation de l'usine.

M. BALLADE a parlé de la nature et de l'évolution des ridens en Loire-Maritime, à la lumière de sondages pratiqués par le Service des Ponts et Chaussées; des résul tats nouveaux ont été donnés sur le cheminement de ces ridens et leurs rapports avec le charriage et le transport de sables en conduites. Ce sujet est très intéressant: plus développé il pourra faire l'objet d'une prochaine communication au Comité Technique.

\section{Commission pour l'inventaire des ressources hydrologiques}

Le 20 janvier $M$. Giguet a exposé devant cette Commission les études en cours à Electricité de France, pour l'inventaire des ressources hydro-électriques industriellement exploitables : à partir d'un seuil de rentabilité, les projets sont classés d'après leur valeur industrielle et le potentiel hydraulique industriel se chiffre ainsi a 87 milliards de $\mathrm{kWh}$ pour la France métropolitaine.

M. Caquot a calculé de son côté l'énergie naturelle, en partant de considérations géographicques et de données hydrométriques; il aboutit à un potentiel ẻnergétique brut de 300 milliards de $\mathrm{kWh}$, dont il estime $1 / 3$ environ comme pouvant être équipé; il rejoint ainsi sensiblement le chiffre donné par $M$. Grouer.

M. SERra indique, d'une part, la méthode appliquéc par l'O.E.C.E. (Commission Economique de l'Energie, Genève) à partir des précipilations et par sommation des produits élémentaires des volumes d'eau écoulés par les altitudes correspondantes; et, d'autre part, une méthode d'évaluation de l'énergie brute d'après les différences des debits à diverses cotes du lit des cours d'eau. ef les dénivellations. Les résultats obtenus sont comparables entre eux et avec les précédents (M. Giguer ef M. Gaduot). 
M. SuTER a présenté un graphique «hydropotentiel» du bassin du Var.

La Commission a conclu que toutes ces méthodes pouvaient coexister : elle examinera en temps opportun l'éventualité d'une prochaine réunion pour prendre connaissance des résultats complets de l'étude en cours ì Electricité de France.

\section{Sous-Section de Glaciologie}

A la réunion du 6 février 1953, la Sous-Section de Glaciologie a entendu, sous la présidence de M. Messines DU Sourbrer, les exposés de :

- MM. Gherrey et Garaver, sur l'ensemble des résultats de leurs observations au glacier de Sarennes, depuis 1949;

- M. Rexnaum, sur la situation de la Mer de Glace en 1951 et 1952 ;

- M. Trucart, sur l'enneigement dans les Vosges (étude de M. Juiltard);

- M. PÉGur, sur un projet de voyage glaciologique des géographes français en Islande, en 1954.

Le projet de I'U.G.G.I. sur la elassification de la neige a été examiné.

Une abondante littérature étrangère a été analysée el on a jeté les bases de la «Tournée Glaciologique»1953, qui aura lieu en Suisse dans les Alpes bernoises.

Il ressort des diverses études glaciologiques présentées ou analysées que les glaciers ont généralement tendance à se réduire, sauf quelques exceptions en Amérique du Nord.

\section{Code d'essais des installations hydrauliques}

M. Escande a réuni le 11 février 1953, la Commission de Révision du Code d'Essais : le chapitre relatif aux déversoirs a été relu et adopté moyennant quelques retouches.

Prochaine réunion le 24 mars pour l'examen de la fin du fascicule 6 , "Caractéristiques de fonctionnement des machines hydrauliques $\sharp$ notamment les normes rela* tives aux pompes.

\section{Commission pour l'étude de la sédimentation}

Au cours d'une deuxième réunion les 25 et 26 février 1953 , cette Commission, présidée par M. Nuzery, a entendu les exposés de spécialistes susceptibles d'orienter les recherches des hydrauliciens sur la sédimentation des réservoirs et des cours d'eau :

M. Hénin, sur la chimie et la structure des minéraux argileux fins et les phénomènes de floculation.

M. Ricard, sur la rhéologie des sédiments.

D'autre part, M. BLANcher s'est attaché à situer le point extrême des études de la sédimentation en laboratoire; M. Bonnin a rendu compte des mesures sur les courants de densité au Sautet en 1952 et M. Mrchon a parlé de ses essais sur la consolidation des vases et sur les courants de densité en canal au Laboratoire National d'Hydraulique (Chatou).

D'intéressants échanges de vue ont suivi ces exposés.

Un schéma sommaire de classification des matériaux a été adopté et sera précisé par la suite.

\section{Commission du déficit d'écoulement}

La Commission du Déficit d'Ecoulement a entendu le 17 mars, sous la présidence de M. Serra, les remarques de M. Coutagne sur les formules de déficit d'écoulement et un exposé de M. PÉguy sur la méthode Thornthwaite (évapo-transpiration).

\section{Publications de la Société}

- Annuaire Hydrologique de la France, 1951, paru en mai 1953;
- Mémoires et Travaux, $\mathrm{n}^{\circ} \mathrm{I}-1953$, paru en juin 1953 ;

- Transport Hydraulique et Décantation des Matérian.x solides iCompte rendu des 2es Journées de l'Hydraulique), paru en juillet 1953;

- Stations Hydrométriques Françaises (3" partie: Pyrénées), en préparation.

\section{COMMUNICATIONS}

\section{AMÉNAGEMENTS HYDRAULIQUES EN U.R.S.S.}

Cette communication n'a pu être présentéc par suite d'un empêchement de M. Tricart.

M. le Président donne la parole à M. Delatrre pour l'exposé de sa communication :

\section{DISPOSITIF DES ENTRÉES DU CANAL. DE NAVIGATION ET DU CANAL USINIER DE DONZËRE-MONDRAGON CONSÉQUENCES SUR LA RÉPARTITION DU DÉBIT SOLIDE}

\author{
Résumé
}

I

L'implantation de la prise d'eau de DonzèrewMondragon sur «l'extrados» de la berge convexe du Rhòne, peu à l'aval du Pont de Donzère, est favorable à la réduction du débit solide dans la dérivation, par suite de la prépondérance des débits liquides du côté de l'extrados. Mais cette ségrégation due à l'aceroissement des vitesses ne se fait sentir qu'en pleine courbe alors qu'il convient pour la navigation que l'entréc du canal se trouve à lamoree amont de la courbe, en prolongement du chenal navigable.

La C.N.R. a ainsi été conduite à envisager deux entrées distinctes, l'une constituant la passe navigable et l'autre la passe usinière.

La passe navigable implantée á l'origine amont de la courbe du fleuve ne s'engrave pas grảce à une surélé vation de son seuil au moyen de palplanches et a l'effet de dégravement obtenu devant la passe usinière at qui se fait sentir à l'amont.

C'est en effet sur la passe usinière, dont le débit rapporté au mètre de seuil est triple de celui de la passe navigable, que sont reportées les difficultés dues à l'engravement: malgré sa position favorable, et en raison même des fortes vitesses qui s'y produisent, il est nécessaire qu'elle se débarrasse automatiquement d'un charriage qui commence à se faire sentir pour des debits du fleuve de $2.000 \mathrm{~m}^{3} / \mathrm{s}$ et dont la ségrégation naturelle est modiffée par suite de l'importance du débit dérivé $\left(1.500 \mathrm{~m}^{3} / \mathrm{s}\right)$.

On a donc cherché une forme de seuil qui :

- D'une part, crée un écoulement suffisamment tourbillonnaire pour remettre en mouvement les matériaux qui tendraient à se déposer au droit de la prise;

- D'autre part, rejette les matériaux en mouvement vers le centre du lit.

Les conclusions des essats sur modèles réduits exécu tés d'abord au Laboratoire d'Fssais Hydranliques de la C.N.R. à Lyon (échelle 1/200), puis at Laboratoire National d'Hydraulique de Chaton (echelle 1/60), sont les suivantes : 
a) Un seuil rectiligne constitué par un mur vertical en béton amorce un rouleau d'axe horizontal qui se propage transversalement, les filets liquides à trajectoire hélicoüdale entrainant les giaviers vers le large; mais cette disposition devient insuffisante dès que le débit dépasse $2.500 \mathrm{~m}^{3} / \mathrm{s}$ et le seuil s'engrave rapidement;

b) Un encorbellement, ajouté au seuil précédent, protège le roulea d'eau des perturbations dues à l'écoulement de surface et favorise son alimentation, sans toutefois maintenir suffisamment son efficacité;

c) Un seuil en dents de scie (pas : $24 \mathrm{~m}$; profondeur : $5 \mathrm{~m}$ ) substitué au seuil rectiligne rénéchit le conrant et favorise la translation du rouleau; en outre, aux points saillants, il se produit une perturbation d'axe vertical qui remet en mouvement les matériaux qui auraient tendance à se déposer.

C'est ce type de seuil, complété par un encorbellement (b) qui a été définitivement retenu : pour une crue de $6.150 \mathrm{~m}^{3} / \mathrm{s}$, de durée normale, avec alimentation en matérianx charriés de $1.500 \mathrm{~m}^{3 / 3} / \mathrm{h}$, il se dégrave automatiquement dans le moindre temps et reste très largement dégagé après le passage de la crue.

II est évident que cette entrée noest pas propice à la eirculation des bateaux.

\section{II}

Si es dispositions donnent satisfaction pour le canal d'amenéc, clles affectent l'équilibre du fleuve puisque le rapport entre le débit solide et le débit liquide se trouve assez notablement augmenté dans le Rhône mort 1.

D'après le Laboratoire National de Chatou, le débit solide roulé, déterminé par les formules du Professeur Meyer-Peter est d'environ 1.700 .000 tonnes par an.

Tout ce débit solide devrait passer par le Rhône mort qui, d'après les mèmes formules et sans modification du lit ni de sa pente, n'est capable de chartier que 300.000 tonnes. On peut cependant espérer que ces chiffres constituent des limites thériques défavorables.

Notamment, le pouvoir d'entraînement du Rhône mort sera augmenté en cas de crues par ouverture totale du barrage de retenue. Des chasses peuvent en outre etre effectuées sans vider le canal, à condition de fermer complètement les barrages de garde placés sur les entrées.

D'autre part, on peut admettre un léger exhaussement du lit a l'aval du barrage de retenue et un certain abais sement à l'aval de la restitution, donc une petite augmentation de la pente du Rhône mort qui provoquera une légère amélioration de la puissance de charriage.

Au total, il est envisagé de ne draguer au début de l'exploitation que le liers du débit solide annuel évalué, soit environ $400.000 \mathrm{~m}^{3}$ par an, étant entendu que l'on surveillera l'évolution du lit: suivant les phénomènes observes, on augmentera ou on diminuera les dragages. En dehors des périodes de crues, on pourra aussi enlever à sec les graviers qui s'accumuleraient dans le Rhône mort.

Mais il faut en ontre tenir compte des risques d'érosion à l'aval de la restitution, risques d'autant plus sérieux qu'à $2 \mathrm{~km}$ plus à l'aval le fleuve est coupé par une barre rocheuse (seuil de Saint-Etienne-des-Sorts), qui n'est recouverte que de $0,50 \mathrm{~m}$ de gravier. En cas de difficultés, on pourra recourir aux moyens ci-après :

a) Pavage du lit à l'aval du seuil de Saint-Etienne-desSorts, de manière à éviter que l'érosion augmente l'importance de ce seuil jusqu'à un point critique pour Ia navigation;

b) Construction en travers du lit, à l'aval du seuil, d'un certain nombre de tenons en enrochements qui fixeraient provisoirement le niveau du fond;

c) Construction, entre Pont-Saint-Esprit et la restitution, d'épis resserrant le lit de manière à provoquer un chariage plus intense et à mobiliser des grat viers vers l'aval;

d) Si tout cela était insuffisant, coupure de la bouele du Rhône mort, immédiatement en amont de la restitution; on aurait ainsi pendant un certain nombre d'années un entrainement important de graviers vers l'aval;

e) Enfin, le seuil rochenx de Saint-Etíenne-des-Sorts no se prolongeant pas sur la rive gauche du fleuve, il serait relativement aisé de creuser une courte dérivation navigable contournant le seuil.

D'autre part, l'aménagement de la chute aval que la C.N.R. effectuera sans doute avant que les inconvénients ci-dessus aient atteint une importance critique, comporto le prolongement du canal de restitution de Mondragon. en dehors du lit du Rhône; à partir de ce moment, la présence du seuil de Saint-Etienne-des-Sorts ne sera plus à prendre en considération.

\section{Commentaire de $M$, le Président :}

M. le président remercie $M$. Delatrare et le félicite de sa promotion dans la Légion d'honneux. Le mémoire de M. Denattre, exposé d'une façon brillante, montre d'une part les difficultés naturelles qu'il a rencontrées, d'autre part l'aide qu'il a trouvée dans les Laboratoires pour la réalisation des ouvrages grandioses de DonzèreMondragon.

La séance est levée à 11 h. 30 .

\section{SEAANCE DU MERCREDI APRES-MIDI 18 MARS 1953}

La séance est ouverte à $14 \mathrm{~h}, 15$, sous la présidence de M. Aillenft.

\section{COMMUNICATION DE M. SERRA}

\section{LES ÉTUDES HYDROLOGIQUES SUR LA HAUTE-DURANCE}

Le mémoire de M. Serra et la discussion ayant suivi sa présentation sont publiés pages 726 et suivantes du présent numéro.

1. Nous appelons thône mort la partie du Hhône situé entre l'entrée et la restitution de la dérivation. Cette expression commode n'est pas tres juste puisque cette partie du Rhone foule toutes les eaux surabondantes non derivées, notamment ecoule toutes les eaux surabondantes non dorives, notamment
toutes les aves. Mais la navigation n'emprumte plus cette toutes les erues.
partie du fleuve.

\section{COMMUNICATION DE M. ROULLEAU}

\section{LA PLUIE ARTIFICIELLE}

Ce mémoire sera publié in extenso dans un prochain numéro de La Houille Blanche.

\section{Résumé}

Mécanisme de la pluie naturelle:

Les averses, comme les pluies continues ne peuvent se produire que si les nuages qui leur donnent naissance sont le siège de mouvements ascendants.

Pour qu'il plenve, il faut, parallèlement à l'ascendance, un état physique du nuage : la coexistence d'eau 
surfondue et de slace, ou encore de gouttes salines hygroscopiques et d'eau.

\section{Formation artificielle de la pluie:}

Le procédé consiste à créer cette deuxième condition (cristaux de glace ou noyaux de chlorure de sodium) qui n'existe pas toujours, $\mathfrak{c}^{3}$ est-à-dire à ensemencer :

- Soit un nuage surfondu, avec des particules très froides (carbo-glace, anhydride carbonique solide, air liquide) ou avec de l'iodure d'argent qui cristallise à très peu près comme l'eau;

- Soit un nuage dont la température est partout supérieure à $0^{\circ}$, avec du chlorure de sodium pulvérisé.

\section{L'ensemencement du nuage se fait}

-- Soit en lâchant sur le sommet du nuage, d'un avion qui le survole, une petite bombe dont l'explosion à l'intérieur du nuage disperse quelques centaines de grammes de neige carbonique;

- Solt en lançant, à partir du sol, des fusées qui éclatent sous le nuage, dispersent de l'iodure d'argent en très fins cristaux emportés au sein du nuage par les courants ascendants.

Daus l'ensemble, les expériences s'accordent pour montres que la pluie artificielle n'est possible qu'en attaquant des cumulus très développes verticalement et en cours de développement: l'insémination dissipe les nuages en voic d'affaiblissement (nuages stratifiés).

\section{Résultats quantitatifs}

Pour des cumulus de $3.000 \mathrm{~m}$ d'épaisseur, les quantités d'eau obtenues sont de l'ordre de 5 à $10 \mathrm{~mm}$ de hauteur. Les abaques de R. Exraun indiquent la hateur de pluie d'après l'altitude de la base du nuage et celle de son sommet ainsi que Ia température à l'un de ces deux niveaux: l'accord avec les observations est bon pour les cumulus ayant atteint leur développement maximum.

La pluie artificielle ne présente d'intérêt pour l'agriculture qu'en période de sécheresse : ainsi pour la Beauce, où la hauteur annuelle de pluie naturelle est de $600 \mathrm{~mm}$, on pent estimer cet apport supplémentaire a $100-150 \mathrm{~mm}$, soit $25 \%$. Mais ces 100 ou $150 \mathrm{~mm}$ exigeraient une centaine de jours d'expérience répartis principalement de mars à septembre. Si l'on admet que les frais de fonctionnement de chaque expérience sont de l'ordre de $40.000 \mathrm{~F}$, et que la surface de précipitation soit a chaque fois de $100 \mathrm{~km}^{2}$, le mi d'eau reviendrait a 25 centimes.

\section{Conclusions}

La pluie artificielle peut etre obtenue dans les cas ci-après :

a) Lorsque Ia pluie naturelle n'a pas encore commencé, mais tomberait d'elle-même quelque temps plus tard : intérêt d'opportunite;

b) Pour provoquer une pluie qui ne se serait pas produite d'elle-même par suite, par exemple, de la limitation du nuage en altitude.

D'autre part. il est possible de faire disparaitre un brouillard et de dégager une piste d'aérodrome en dissipant un nuage, ou de dissociex un nuage givrant pour améliorer les conditions de yol.

Enfin, on peut éviter la formation de la grêle, en hâtant l'évolution d'un cumulo-nimbus, mais il convient d'être prudent sur ce dernier point, afin de ne pas provoquer le phénomène inverse.

De toute façon, jusqu't maintenronl, il ne paraît pas possible de modifier, dans quelque sens que ce soit, les précipitations d'un système nuageux de grande perturbation. On ne pent songer qu'à agir sur les nuages d'averses, donc à ne modifier que partiellement le régime pluviométrique dans les régions temperces tout au moins.

\section{Discussion}

M. le President Anlener remercie M. Roulleau de sa clare et intéressante communication, ef se plait a montrer qu'il existe encore, dans la science, des domaines tels que celui de la pluie artificielle, ou la passion de la recherche naît de la primeur du problème et croil par la possibilite de son évolution rapide.

D'autre part, M. le Président rappalle que les premières et récentes publications des Américains sur cette question ont mis lacent sur certains effels à grande distance et à retardement des essais de précipitalions artificielles, effets révélés par ume hausse de la pluviométrie les dimanches, dans l'Est des Etats-Unis, alors que les ensemenceurs de nuages travallent principalement dans louest et se reposent sans doute le dimanche. La General Electric Company a décidé, de ce fait, de ne pas poursuivre ses expériences de pluie artificielle tant qu'une législation du procédé ne la mettrait pas a l'abri de réclamations motivées par d'eventucls «effets a dis* lance» qui pourraient être imputés à ses essais.

M. Roulteau explique, en réponse à M. le Président, que les eristaux d'iodure d'argent employés pour l'ensemenement des nuages, peuvent du fait de leur tress petite dimension, suive un trajet considerable a partir de leur point d'émission, el influencer des nuages tres éloignés. Cependant, Schaefren, qui tmaille pour la G.E.Co., a montré que ces cristaux devenaient inactifs au bout d'un temps extrêmement court ct cela exclurail leurs effets à distance. D'autre part, les périodicités, notamment celles de sept jours, ne sont souvent quapparentes en météorologie.

M. de Montmanun signale que la périodicité des précipitations, à laquelle fait allusion M. le Président, el sa corrélation avec les essais de plute artifeiclle, onl dé contestées par certains auteurs américains.

M. DE Montmarin fait part du résultal d'essais de pluie provoquee entrepris en Tunisie. Des moyens tres divers ont été employés (neige carbonique, solution saturée de différents sels, mèches à iodure d'argent, etc.); sauf exception les résultats oblenus ont eté quantitativement assez faibles et ont montré la diffenlés de localiser l'effet dans l'espace. On a tendance actuellement à abandonner l'attaque des nuages à partir d'avions ef opéres à partir du sol soit par fusées, soit par générateurs fixes disposés en des points où existent des courants ascendants; des essais de ce genre ont déjà été effectués en Algérie et le seront hientôt en Tunisic.

M. Ch. BEAU demande d'abord s'il suffit d'altaquer le nuage en un point pour provoquer sa condensalion totale, puis si la durée limitée des nuages est compatible avec la possibilité d'action à distance, mentionnée par M. AHLFHET.

M. RoullaA répond : a la première question que la précipitation provoquéc en un point s'étend lrès rapide. ment (vitesse apparente de propagation des cristaux : $60 \mathrm{~km}$ à l'heure) et se généralise à l'ensemble d'un nuage camuliforme; à la deuxième question, qu'il existe dec «cellules» particulièrement visibles par temps d'orage. à linterieur desquelles un nuage ayant accompli son cycle disparait, mais est remplace presque aussitot pat un autre, qui prend naissance en un point voisin, ef ainsi de suite, de sorte que pour un observateur non avisé un cumulomimbus paraît généralement subsister plus d'une beure ou deux.

M. Duffavt demande s'il a te fait des essais de pluic artificiclle en vue de la production d'énergie hydraulique

M. Ie Président ne le eroit pas, ear lhydroélectricile est tout de même moins intérossce à l'abondance de 
l'enu que l'agriculture l'est à la production de la pluie, la viticulture à la suppression de la grêle, et, surtout, la navigation aćrienne à l'absence de brouillard. D'ailleurs, le problème vu sous l'angle de l'énergie hydro-électrique se poserait dans des conditions beaucoup plus précises qui comporteraient notamment non seulement la production de la pluie, mais encore le contrôle de cette production : un schéma de ce contrôle, envisagé il y a quelques années avec M. Roulleau, dans un polygone de $100 \mathrm{~km}$ de rayon, la Beauce, par exemple, nécessiterait des milliers de pluviomètres à transmission à distance centralisée, en même temps et au même point que le contrôle des avions émetteurs de cristaux pluviogènes. Le coût d'un tel équipement atteindrait 100 à 200 millions, seulement pour le polygone d'essai; on peut espérex que les specialistes de la navigation aérienne, plus intéressée à la question que le sont les hydro-électriciens à mettre un peu plus d'eau dans les réservoirs. pourront faire l'effort financier correspondant à cet essai.

M. DE Montmarin signale les expériences de M. Dessens dans les Pyrénées, dont M. le Président précise qu'elles étaient destinées à la recherche des conditions de formation de la pluie à l'intérieur d'un nuage, mais non à la production de pluie artificielle.

M. le Président insiste sur le fait que la plupart des techniques, et notamment l'hydro-électricité, en sont présentement à chercher à comprendre le phénomène de la pluie artificielle plutôt qu'à chercher à l'exploiter.

M. Esclangon se demande si les diverses économies groupées pour l'aménagement de la Durance ne seraient pas intéressées par la réalisation de tels essais.

M. le Président pense que la situation et l'orographie de ce bassin rendraient l'essai initial moins commode qu'en Beauce, où la régularité du relief et des courants atmosphériques faciliteraient les expériences et les observations.

M. Serra indique qu'on pourrait penser au procédé pour la production de crues artificielles dans les bassins expérimentaux.

M. Mordiltat demande comment se répartissent sur la Beauce les $600 \mathrm{~mm}$ de pluie annuelle moyenne pendant les cent jours où elle tombe, et si la proportion est plus forte dans la période où elle est le plus utile à l'agriculture, c'est-à-dire, entre le 15 juin et le 15 septembre.

M. Rouleav indique que le nombre moyen mensuel de jours de pluie est d'une dizaine en été et de quinze environ en hiver. Il ajoute que le procédé de pluie artificielle peut être intéressant pour l'agriculture, dans le cas, assez fréquent, où, les nuages se développent verticalement beaucoup, créant, par conséquent, les conditions favorables à la pluie artificielle, mais se trouvent bloqués a certain niveau par une couche d'inversion gênant le mécanisme de la pluie naturelle.

M. Mordrulat expose que ce régime nuageux semble être celui que l'on peut rencontrer en bordure d'anticyclones, sous des pressions peu élevées en périodes extrêmement sèches, comme cela est arrivé en 1947 et 1949 , d'où l'intérêt du procédé exposé.

M. Monlat demande si des considérations générales sur le mécanisme d'humidification de l'atmosphère ne permettent pas de déterminer si, à longue échéance, l'humidité fournie à l'atmosphère ne seraít pas constante, de sorte que le déclenchement artificiel de Ia pluie ne reviendrait qu’à déplacer dans l'espace et dans le temps les précipitations.

M. Roulleat répond en distinguant, en matière de pluie, la grande perturbation prépondérante en Europe et sur laquelle le procédé n'a aủcun effet et la pluie d'averse dont le déclenchement artifieiel est très localisé : ii est possible, par exemple, ru'une pluie destiné à Etampes tombe axtificiellement à Orléans, mais à cette échelle, la diffusion atmosphérique est si grande qu'll est peu probable qu'on prive réellement d'eau un point; au moment ou la pluie tombe, il $y$ a évaporation d'une partie de l'eau précipitée qui est ainsi remise dans la circulation atmosphérique.

M. Morlat remercie M. Rovlleav et précise qu'il a posé cette question pour s'assurer que la possibilité d'augmenter de $150 \mathrm{~mm}$ la pluie annuelle sur la Beauce, signalée par le conférencier, n’était pas extensible à une région plus vaste.

M. Roulleau est bien de cet avis, et précise que l'intérêt de la pluie artificielle est de localiser les averses dans le temps et dans l'espace.

M. De Montmarn signale que les auteurs des essais tunisiens se sont posé la question de savoir si l'intervention artificielle sur les nuages augmentait réellemeni les précipitations totales, ou ne faisait que changer leur répartition. Dans certains cas particuliers, l'étude du régime des vents peut donner des indications utiles : c'est ainsi qu'en Tunisie la majeure partic des précipitations paraît être amenée par le vent du nord-ouest qui vient du bassin occidental de la Méditerranée, traverse la Tunisie en diagonale et, après avoir franchi les hauteurs de la Dorsale, se dirige vers le golfe de Gabès. II est certain que les nuages qui franchissent la côte est de la Tunisie en direction du large sont perdus pour tout le monde. D'où l'intérêt de les « cloner au sol » avant qu'ils aient atteint la côte.

COMMUNICATION DE M. PARIEE

\section{REMARQUES SUR L'ÉVALUATION DES TRÈS GROS DÊBITS DES RIVIËRES EN FONCTION DES HAUTEURS D'EAU, A LA LUMIÈRE DE RÉCENTS JAUGEAGES DE GRANDES CRUES}

\section{Résumé}

Un des problèmes qui intéresse le plus les hydrolognes, mais aussi leur apporte très souvent de grands embarras, est la connaissance des débits qui correspondent aux niveaux maxima des grandes crues. Car dans la plupart des pays, l'immense majorité des rivières, y' compris les principales, n'a été l'objet de mesures directes que pour des cotes très inférieures à leurs records. Les courbes des débits en fonction des hauteurs ne sont done expérimentales que dans les parties basses et moyennes des tracés. Pour des valeurs plus fortes que celles des petite crues ou, en certains lieux déjà privilégiés, de crues assez considérables mais point extraordinaires, les courbes de tarage doivent être extrapolées. Pour guider ces prolongements, il existe des méthodes relativement satisfaisantes : calcul des débits maxima au moyen de formules (pente-rugosité on dénivellation brusque avec $\sqrt{2 g h \text { ) }}$ transposition, addition, soustraction de débits connus avec une exactitude suffisante pour l'amont on l'aval, comparaison et mise en concordance des volumes liquides totaux a des stations voisines, confrontation de ces cubes avec les pluies, etc. Mais pour beaucoup de tronçons fluviaux, les bases numériques indispensables pour ces calculs sont absentes ou trop peu nombreuses et pen ou point surres. Done, fort souvent, on extrapole par des procédés de fortune, ou plutôt d'infortune. On rallonge la courbe par l'équation de sa partie connue, ou par la 
tangente à l'extrémité supérieure de ce segment, on bien par une ligne incurvée qu'on trace au sentiment, pour ne pas dire au hasard.

En effet, la tangente est en principe fausse parce que laccroissement ordinaire de la section mouillée et de la vitesse pour des eaux montantes fait de la courbe normalement une parabole convexe vers l'axe des hauteurs Mais l'équation de la partie bien connue ne vaut que si. pour des niveaux supérieurs, la pente, la rugosité générale ne changent point, ou ne se modifient que selon les taux applicables aux cotes plus basses et si la section mouillée ne se dilate pas, plus ou moins brusquement, de manière a comporter un champ d'inondation sur lequel peuvent passer des débits notables. Dans ce cas, au-delà d'un certain niveau, la courbe se redresse ver's le haut, bien plus que ne le voudrait l'équation de son tracé inférieur (voix plus loin des exemples).

Au contraire, si la rugosité du lit augmente dans lo haut des berges, ou si un rétrécissement marqué à l'aval diminue la pente au lieu de l'échelle, à partir de certains niveaux, l'équation de la partie basse donnera des débits trop forts; il faudra continuer la parabole par une courbe moins concave (Rhin à Bâle) ou mème par une tangente ou par un tracé presque rectiligne (Rhin à Rheinfelden); et enfin, lorsque le rétrécissement d'aval exerce une action extrèmement sensible, par une courbe qui tourne sa concavité ver's l'axe des hauteurs (Inn à Schärding certainement, Agout à Lavaur et Ardèche à Vallon, très probablement). Mais c'est le phénomène inverse qui se produit et le dessin contraire qui s'impose, lorsque le resserrement, exagérant les hauteurs à son amont ou dans un parcours en gorge, accentue la pente superficielle à sa sortie ou à l'aval immédiat, ou même dans l'étroit lui-même s'il n'est point trop long. Quand cet effet devient bien plus décisif que pour des cotes plus basses, la courbe se redresse vers le haut et indique un pen comme s'il $y$ avait inondation (avec bien moins d'écart cependant) des débits supérieurs à ceux de l'équation de la partie basse et moyenne (Ardèche inférieure au débouché de sa gorge à Saint-Martin, Saône à Lyon). Par malheur, à moins de se fonder sur l'expérience, i] est souvent difficile de connaittre avec précision quels phénomènes modifient les conditions d'écoulement en très grandes crues; et même si on a pu les déterminer, il reste encore fréquemment aventureux de vouloir chiffrer linfluence de ces facteurs sur les relations entre cotes et débits.

La seule méthode sûre pour l'extrapolation des courbes ici considérées est le recours à des jaugeages de grandes crues, opérations très rares ainsi que nous l'avons dit au début de ce texte. Encore faut-il que ces mesures, difficiles en maints cas, aient étè pratiquées avec une technique satisfaisante. Sans quoi, au lieu d'erreurs possibles mais tolérables de 5 à $10 \%$ dans un sens on dans lautre, qui menacent les résultats des jaugeages les meilleurs de grosses eaux, on peut avoir des inexactitudes systematiques on aberuntes plus fâcheuses; el alors l'extrapolation peut pécher autant que si on l'avait conduite en utilisant des valeurs non expérimentales. Et l'on a lieu de croire que les résultats de nombreux jaugeages anciens effectués avec un outillage moins perfectionné que celui de maintenant, appellent des correctifs. La discordance des indications avec celles de mesures récentes peut cependant avoir pour causes des changements dans les sections mouillées, les pentes, etc., par suite de creusements, de remblaiements, de croissance végétale, de travaux humains, etc.

Or, des jaugeages de débits élevés, quoique généralement très inférieurs aux records, ont eu lieu récemment en France. Et aux Etats-Unis, on a mesuré, grâce a de rrais tours de force, depuis une vingtaine d'années, maints débits tout it fait exceptionnels. Nous donnerons quelques renseignements brefs sur certaines de ces experiences et sur les conclusions à en tirer.

Fn décembre 1952, M. Barriène, Ingénicur T.P.F. à la
$4^{4}$ circonscription électrique de Limoges, a mesuré par moulinet en toutes profondeurs sur la Dordogne a Saint-Seauves, $1.042 \mathrm{~m}^{3}$, et sur la mème rivière, $1.920 \mathrm{mb}^{\mathrm{b}}$ ¿ Domme et 2.317 a Bergerac. Malgré la nécessité d'un porte à faux étendu, ce résultat ne contredit point $t$ priori nos évaluations précédentes qui attribuaient 3.500 a $4.000 \mathrm{~m}^{3}$ à la crue de décembre 1944 et au moins 5.000 aux flots exceptionnels de janvier 1728 et mars 1783. En revanche un jaugeage fait le 21 décembre 1952 sur le Cher à Selles n'a donné que $610 \mathrm{~m}^{3}$, ce qui, sans certitude absolue, rend vraisemblable pow lo record de mai 1856 un dẻbit nettement inférieur à $1.690 \mathrm{~m}^{3}$, chiffre admis précédemment et basé sur des formules. Puis deux jaugeages remarquables de M. Barniene pour la LoireInférieure à Ingrandes, en fonction des hauteurs à l'échelle de Montjean, ont indiqué, pout $5,76 \mathrm{~m}, 3.800 \mathrm{~m}$ et pour $6,15 \mathrm{~m}, 4.730 \mathrm{~m}$, chiffres respuctivement inférieurs de $19,5 \%$ et de $10 \%$ à ceux d'une courbe ablablic précédemment d'après des jaugeages opérés en toutes profondeurs jusqu'à des cotes de 6,26 et $6,32 \mathrm{~m}$. On peul admettre que des changements de fond ont causé ces divergences (à vrai dire lon aurait plutôt tablé antérieurement a ce point de vue sur une influence contraire). Cependant, il est difficile de ne pas supposer qu. pour une cause encore peu discernable, les jaugeages précédents ont exagéré les débits de crue, pour le moins de quelques centièmes. Néanmoins, le chiffre de 6.250 i 6.500 attribué précédemment au maximum connu ne paraît pas devoir ètre modifé sensiblement, car on doil le rapporter non point au niveau réel de $6,78 \mathrm{~m}$, mais i celai de $7,10 \mathrm{~m}$ qui aurait été vraisemblablement atteint sans la rupture des digues.

D'autre part, la Compagnie Nationale du Rhone a jaugé en surface vers son maximum une grande crue du Roubion à Montélimar, en novembre 1951; d'où avec extrapolation de $60 \mathrm{~m}^{3}$, un débit de $590 \mathrm{~m}$; pour la pointe, ce qui concorde avec mes évaluations ancicunes pour les très gros débils (sans indications de probabilite moyenne) de la rivière. Mais certaines etudes donnenl à penser que la crue de juillet 1892 a dé bien plus puissante. Puis la même Compagnio a jaugé sur le thoone a Lafarge, près du Teil, en nov,-déc. 1950, des débils allam jusqu'à $4.173 \mathrm{~m}^{3}$. Ces valeurs sont nettement plus fables que celles qui résultaient d'une courbe precédente qui paraissait cependant mériter grande confance. Comme on attribuait $10.000 \mathrm{~m}^{3}$ a la plus grande crue connue, l'extrapolation nouvelle qui reste a faire expose at des conclusions très incertaines. Cependant, il devient probable que les plus gros débits de 1840 et 1856 n'ont pas en ce point dépassé 7.500 à $9.000 \mathrm{~m}^{3}$. Et provisoirement on admettra 8.000 a $8.500 \mathrm{~m}$. Puis des mesures faites par la Compagnie sur le Rhône supénieur à Sault-Brénaz oủ la courbe semble devoir etre prolonge aumdela de $1.335 \mathrm{~m}^{3}$ par l'équation de sa partie basse, impliquent très probablement une sensible erreur par exces des calculs précédents. Il devient raisonnable d"admetlre $2.500 \mathrm{~m}^{3}$ an lien de 2.800 ou 2.900 pour les records (1856 et 1944). En revanche les jaugeages complets (avec exicution trés difficile vu la violence des courants) de devx grandes crues non extraordinaites sur la Durance h Mirabeau en novembre $1951\left(2.750\right.$ et $\left.2.860 \mathrm{~m}^{3}\right)$ coincident à peu près avec mes estimalions précédentes pour les hauteurs en question et ne paraissent point du tout. incompatibles avec les $5.000 \mathrm{~m}^{3}$ ou un peu plus admis par moi pour les crues execptionnelles (novembre 1843 et octobre 1882). Mais en ce lieu resserre, les niveaux du fond et les pentes sont très variables; de toutes façons, on fera bien de se horner a admettre jusqu'a plus ample informé, pour les records du $x x^{4}$ siecle, 4.000 â $6.000 \mathrm{~m}^{3}$.

L'ensemble do ces résultats pour la plupart des rivieres franģaises considérées, aboutit à une réduction de 5 i $20 \%$ des chiffres anterienrement admis. Mais on aurat tort de croire que partout les evaluations anciennes ont exagéré les debits des crues cxiraordinaires. 
Lil certitude que des crrcurs inverses parfois très graves sont aussi à craindre nous est donnée par de remarquables jaugeages récents sur diverses rivières des EtatsEnis. Par exemple, los valeurs formidables 19.000 it $20.000 \mathrm{~m}^{3}$ assignées aux maxima de la Susquehanna inférieure ont été reconnues exactes grâce aux mesures de Inar's 1936 à Harrisburg pour une crue record $(20.900$ et 22.300 à Marietta). Et lors de la même crue exceptionnelle, on a trouvé pour le Potomac à Washington un débit très largement supérieur $(13.700$ contre 9.000) à celui qu'on avait calculé par formule pour le maximum à peu près identique de juin 1889 . Et, fait bien plus saisissant. des jaugeages opérés lors de la catastrophe de juillet 1951 dans les lits principaux of sur les champs d'inondation pour la Kansas River à Topeka ef Kansas City, pais sur la Neosho à Parsons, ont indiqué des débits supérieurs de 30 à $40 \%$ peut-ôtre à ceux qu'on aurait attendus d'après les extrapolations précédentes. Ces expéricnces ont encore montré que les prolongements simples d'après les équations des parties basses au-delà des cotes de débordement auraient suggéré des débits trop faibles de 50 à $60 \%$ pour la Neosho et de 40 à $50 \%$ pour la Kansas à Topeka. Cela tient au fait que de très fories proportions desdits flots $(10.500$ sur 11.600 de la Neosho et plus de 7.500 sur 13.240 de la Kansas à Topeka) passaient dans les champs d'inondation. A ces deux postes, les deux combes de débits se redressent teriblement vers le haut $a u$-dessus de $6,50 \mathrm{~m}$ a $7 \mathrm{~m}$.

Ces exemples semblent confirmer, non certes les valeurs, mais les allures des extrapolations que j'ai faites en employant dans la mesure où je l'ai pu les meilleures méthodes, pour les courbes des débits de la Garonne à Agen et Tonneins, au-delà de $7 \mathrm{~m}$ et $7,50 \mathrm{~m}$, niveaux à partir desquels commencent les inondations, profondes de 2 a $4 \mathrm{~m}$ sur de grandes largeurs $(2$ à $3 \mathrm{~km}$ ) lors des crues les plus puissantes du fleuve. J'ai développé tous ces points dans un texte dactylographié d'une soixantaine do pages. La Société Hydrotechnique de France en possède un exemplaire que l'on peut consulter. J'espère pouvoir adjoindre à ce mémoire les tirages d'une vingtaine de figures.

\section{Discussion}

M. le Président remercie M. PAndé et le félicite d'avoir permis par un enregistrement fidèle d'un grand nombre de chiffres de débits et de hauteurs, de donner une idée précieuse sur la forme des courbes $Q(\mathrm{H})$ et, parallèlement, sur l'évolution du profil transversal des cours d'cau.

M. Morombat demande à M. PARné s'il comnait l'époquo à laquelle le zéro des principales échelles limnimétriques a été fixé lo long de la Loire.

M. Mondrlat pense qu'il faut remonter à la période 1798-1800, le Gouvernement ayant prescrit à l'époque un inventaire général des ressources hydrauliques.

Concernant Saumur, il a retrouvé une indication précise datant de 1838 et donnant l'étiage de 1800 pour origine de l'échelle en ce point. Il pense que la décision a dû être générale et affecter les échelles principales : Nevers, Gien, Orléans, Tours, Langeais, Saumur, Montjean, etc.

$\mathrm{Or}$, on constate actucllement que, pour de mêmes basses eaux, certains étiages tels que ceux de Montjean et d'Orléans diffèrent beaucoup plus que d'autres des zélos des échelles.

Il semble que cet effet n'ait pas pour origine les seuls phénomènes naturels, mais encore et surtout l'intervention humaine : en premier lieu les travaux de protection contre les crues vers $1856-1860$, également les besoins en ballast lors de la construction des chemins de fer; plus recomment, l'aménagement de la Loire maritime et in réparation des dommages de guerre.
On est ainsi conduit a mettre en doute la valeur des débits anciens tirée des courbes actuelles de tarage puisque les profils d'étiage ont considérablement varié.

Il s'ensuivrait que les chiffres donnés par les prédécesseurs de M. Barricire et cités par M. Pards peuvent être parfaitement valables quoique discordants.

M. PandE confirme ce point de vue par le rappel des deux chiffres figurant dans son exposé, pour un même profll transversal du $P \hat{o}$ à Roncocorrente, les sections mouillées élaient de $6.380 \mathrm{~m}^{2}$ en 1874 et $5.834 \mathrm{~m}^{2}$ en 1913 , ce qui prouve que l'historique joue un grand rôle dans l'étude des débits.

M. Mondulat précise - à l'appui - que les débits d'étiage trouvés en 1945,1947 et 1949 sont très voisins de ceux qui ont été trouvés pour les années très sèches de 1870 a 1906 , mais que les hauteurs lues aux échelles peuvent différer considérablement (comme à Orléans) en raison des dragages considérables effectués pour les travaux de reconstruction depuis 1945 .

M. PArné indique que, dans de nombreux cas, le fond se creuse dans une partie et se remblaie dans une autre.

COMMUNICATION DE M. COUTAGNE

présentée par M. Boyen

\section{PRÉVISIONS DES DÉBITS D'ÉTÉ DU HAUT-RHIN (RHIN SUISSE) : ANALYSE DES MÉMOIRES DU Dr HOECK (SUISSE) ET DU Dr CHURCH (ETATS-UNIS D'AMÉRIQUE), ET COMMENTAIRES}

Résumé

\section{PREMTERE PARTIE}

ANALYSE DU MÉMOIRE : Forecasting the summer runoff in the Rhine river. - Nevada agricultural experiment station: M. Church. - Research Bulletin 1 (mémoire principal) and 2 (supplément concernant lo Neckar) :

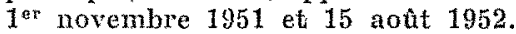

L'objet déterminant du mémoire de M. Church est l'étude des possibilités de prévision des débits d'été du Rhin suisse, compte tenu notamment de l'intérêt que présente cette question pour lo fonctionnement du lac Ijssel (irrigations dans le Zuider Zee). Ellc a été motivée par un rapport présenté à l'Association Internationale d'Hydrologie à Oslo (1948) par le Dr Erwin Hosck, décédé récemment. L'auteur, en rappelant cette étude, et d'autres de Thissse et de Votiken, relatives au lac ljssel, a élargi la question, comple tenu des renseignements complémentaires qu'il a recueillis directement, en Suisse, auprès du Dr Hoeck et autres personnalités.

Ce travail, outre I'intérêt particulier et l'intérêt général qu'il présente, a le mérite de contenir de nombrenx tableaux numériques, à l'appui des considérations ef conclusions de l'auteur, quí constituent une documenfation précieuse en vue d'autres études complémentaires ou rectificatives.

Consmérations générales sun le nassin du Rhin a Ree. (a proximité de la frontière hollandaise)

Ces considérations peuvent se résumer par les donnée. numériques du tableau 1 , concernant les débits et le précipitations du hassin et de ses principanx bassin constituants : le Rhin à Bâle, le Main à Schweinfurt, 1 Moselle a Kochem, et l'ensemble des antres bassins secon daires non étudiés particulièrament (et accessoirement I Neckar).

Toutefois, on doit formuler certaines réserves, en $\mathfrak{c}$ qui coneerne les donnèes pluviométriques dont 
Dr Chunch fait état. Le nombre des stations - au total 13 pour ees bassins de $160.000 \mathrm{~km}^{2}$ - est trop minime. Si, comme nous le verrons, les six stations considérées pour le Rhin à Bâle apparaissent assez représentatives (dans Ieurs variations périodiques annuelles) de la pluviositë, il n'est est pas de même de celles considérées pour les autres bassins qui - même relativement - et surtont en valeurs absolues, demanderaient à être complétées et révisées.

Il suffit de calculer les déficits d'écoulement qui cn résultent, compte tenu des débits, pour $s^{2} e n$ rendro compte (voir dernières colonnes à droite, en bas du tubleau 1).

Quoi qu'il en soit de ces réserves, on peat retenir que le Rhin supérieur (à Bâle) représente, en superficie, $22,5 \%$ et ses débits $43 \%$ des totaux correspondants à Rees.

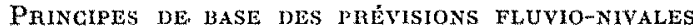

L'auteur considère comme plus efficace la mesure de la neige au sol (efficiency in snow cover) que sa mesure au moment oì elle tombe (nivomètres: defects in snow gage measurements). Il fait elat, notamment, des factenrs secondaires (correction factors), soit de l'état antérieur (lu sol (soil priming) soit des précipitations estivales (summer rains).

De fail, dans le cas considéré de la prévision de l'écoulement du Rhin à son entrée en Hollande, les facteurs dits secondaires apparaissent comme beaucoup plus importants que lo facteur nival propremenl dit : meme pour le Rhin à Bâle, les variations de débit estival dépendent, grosso modo, du facteur nival (précipitations hivernales) pour 50 \% seulement, et pour le reste du bassin (125.000 km² contre 36.000 ì Bâle), le facteur nival ne joue qu'un rôle secondaire 1 .

Aussi, en ce qui concerne plus particulièrement lo bas Rhin, e'est-at-dire a Rees, par exemple, la prévision fe l'econlement estival sort da cadre des prévisions nibo-

1. Ce ròle ne prut cutre precisé qu'en étudiant directernent les variations saisonnieres a Rees, et leur condilionmement par les précipitations de tout le bassin.

\section{TABLEAU I}

DÉbtts. - Bassin du Rhin a ReEs et Bassins constituants principaux (en m":s et en "\%)

\begin{tabular}{|c|c|c|c|c|c|c|c|c|c|c|c|c|c|c|}
\hline \multirow[b]{2}{*}{ Bassins } & \multirow{2}{*}{$\begin{array}{c}\text { Super- } \\
\text { ficie } \\
\mathrm{km}^{2} \\
\mathrm{et} \\
\text { of }\end{array}$} & \multicolumn{13}{|c|}{ DÉnเาง } \\
\hline & & oet. & Nov. & Déc. & Janv. & Fèv. & Mars & Avril & Mai & Juin & Jnill & Août & Sept. & Annee \\
\hline $\begin{array}{l}\text { Rhin a Bale : } \\
\qquad(1808-1947)\end{array}$ & $\begin{array}{l}35.925 \\
22,5 \%\end{array}$ & $\begin{array}{r}921 \\
7,5 \%\end{array}$ & $\begin{array}{r}813 \\
6,6 \%\end{array}$ & $\begin{array}{r}739 \\
6,0 \%\end{array}$ & $\begin{array}{r}669 \\
5,4 \%\end{array}$ & $\begin{array}{r}664 \\
5,4 \%\end{array}$ & $\begin{array}{r}774 \\
6,3 \%\end{array}$ & $\begin{array}{r}975 \\
7,9 \%\end{array}$ & $\begin{array}{r}1.243 \\
10,1 \%\end{array} \mid$ & 12,431 & $\begin{array}{r}1.523 \\
12,4 \%\end{array}$ & $\begin{array}{r}1.342 \\
10,9 \%\end{array}$ & $\begin{array}{l}1.125 \\
9,1 \%\end{array}$ & $100 \%$ \\
\hline $\begin{array}{l}\text { Main-Schweinfurt : } \\
(1925-1947)\end{array}$ & $\begin{array}{l}12.719 \\
8,0 \%\end{array}$ & $6,0 \%$ & $\begin{array}{r}125 \\
9,4 \%\end{array}$ & $\begin{array}{r}114 \\
8,6 \%\end{array}$ & $\begin{array}{r}145 \\
11,0 \%\end{array} \mid$ & $\begin{array}{r}173 \\
13,1 \%\end{array}$ & $\begin{array}{r}174 \\
13,2 \%\end{array}$ & $10,6 \%$ & $6,4 \%$ & $6,1 \%$ & $5,7 \%$ & $5,4 \%$ & $4,6 \%$ & $100 \%$ \\
\hline $\begin{array}{l}\text { Moselle-Kochem : } \\
\quad(1937-1948)\end{array}$ & $\begin{array}{r}28.156 \\
17,6 \%\end{array}$ & $\begin{array}{r}386 \\
8,5 \%\end{array}$ & $\begin{array}{r}465 \\
10,2 \%\end{array}$ & $\begin{array}{r}366 \\
8,1 \%\end{array}$ & $\begin{array}{r}372 \\
8,2 \%\end{array}$ & $\begin{array}{r}534 \\
11,7 \%\end{array}$ & $\begin{array}{c}4.27 \\
9,4 \%\end{array}$ & $7,1 \%$ & $\begin{array}{c}331 \\
7,3 \%\end{array}$ & $\begin{array}{r}316 \\
7,0 \%\end{array}$ & $\begin{array}{r}385 \\
8,5 \%\end{array}$ & $\begin{array}{r}389 \\
8,6 \%\end{array}$ & $\begin{array}{r}252 \\
5,5 \%\end{array}$ & $100^{379} \%$ \\
\hline Autres bassias: & $\begin{array}{c}82.883 \\
51,9 \%\end{array}$ & $\begin{array}{r}904 \\
7,7\end{array}$ & $\begin{array}{r}927 \\
7,9 \%\end{array}$ & $\begin{array}{r}1.221 \\
10,4 \%\end{array}$ & $\begin{array}{r}1.574 \\
13.4 \%\end{array}$ & $\begin{array}{r}1.629 \\
13,9 \%\end{array}$ & $\begin{array}{r}1.585 \\
13,5 \%\end{array} \mid$ & $\begin{array}{r}1.413 \\
12,1 \%\end{array}$ & $\begin{array}{r}621 \\
5,3 \%\end{array}$ & $\begin{array}{r}352 \\
3,0 \%\end{array}$ & $\begin{array}{r}427 \\
3,6 \%\end{array}$ & $\begin{array}{r}458 \\
3,9 \%\end{array}$ & $\begin{array}{r}592 \\
5,1 \%\end{array}$ & $100^{975} \%$ \\
\hline Total à Rees : & $\begin{array}{c}159.683 \\
100 \%\end{array}$ & $\begin{array}{l}3.200 \\
7.7 \% \text { ort }\end{array}$ & $\begin{array}{l}2.330 \\
7,8 \%\end{array}$ & $\begin{array}{l}2,440 \\
8,2 \%\end{array}$ & $\begin{array}{l}2.760 \\
9.2 \%\end{array}$ & $\begin{array}{r}3.000 \\
10,0 \%\end{array}$ & $\begin{array}{l}2.960 \\
9,9 \%\end{array}$ & $\begin{array}{l}2.850 \\
9.5 \%\end{array}$ & $\begin{array}{l}2.280 \\
7,6 \%\end{array}$ & $\begin{array}{l}2.280 \\
7,6 \%\end{array}$ & $\begin{array}{l}2.410 \\
8,1 \%\end{array}$ & $\begin{array}{l}2.260 \\
7,6 \%\end{array}$ & $\begin{array}{l}2,030 \\
6,8 \%\end{array}$ & $\begin{array}{c}2.390 \\
100 \%\end{array}$ \\
\hline Neckar : & 14.000 & & & & 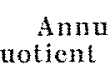 & 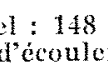 & 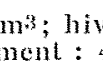 & : & $\%$ & $:$ & $\%$ & & & \\
\hline
\end{tabular}

\begin{tabular}{|c|c|c|c|c|c|c|c|c|}
\hline \multicolumn{6}{|c|}{ Précipitations (en mm et en $\%$ ) } & \multirow{2}{*}{ STATHOSS PJUVIOMËTHQUES (HOMble) } & \multirow{2}{*}{$\begin{array}{c}\text { Débits } \\
\text { en mum } \\
Q\end{array}$} & \multirow{2}{*}{$\mathrm{H}-\mathrm{Q}$} \\
\hline Bassins & $\begin{array}{l}\text { Oct.- } \\
\text { mars }\end{array}$ & $\begin{array}{l}\text { Avril- } \\
\text { sept. }\end{array}$ & Annèe & $\begin{array}{c}\text { Hiver } \\
\%\end{array}$ & $\begin{array}{c}\text { Fité } \\
\%\end{array}$ & & & \\
\hline Rhin supérieur (Bâle)... & 468 & 767 & 1.235 & 37,6 & 62,4 & 6 siations & 902 & 338 \\
\hline Main .. & 457 & 461 & 918 & 50,0 & 50,0 & 1 stalion .... & 272 & 640 \\
\hline Moselle .... & 903 & 782 & 1.775 & 54,7 & 45,3 & 3 stations & 424 & 1.351 \\
\hline \multirow[t]{2}{*}{ Autres bassins } & 725 & 622 & 1.346 & 53,9 & 46,1 & Estimation d'après Main et Moselle. . & 371 & 975 \\
\hline & 596 & 694 & 1.291 & 46,2 & 53,8 & 10) stations pour $160.000 \mathrm{~km}^{2} \ldots \ldots$ & 472 & 819 \\
\hline Neckar $\ldots \ldots \ldots \ldots \ldots$ & 285 & $44 \%$ & 730 & 39,0 & 61 & 3 stations .... & 334 & 396 \\
\hline & & & & & & Friedrich & 302 & \\
\hline
\end{tabular}


fluviales, et doit être abordé et résolu par d'autres méthodes, basées sur d'autres considérations statistiques.

En ce qui concerne la méthode bien connue de M. Church, nous avons eu l'occasion de montrer comment elle pouvait etre améliorée, par la considération, au lieu de la formule de proportionnalité $Q / Q_{0}=\mathrm{N} / \mathrm{N}_{0}$, de la formule, établie statistiquement :

$$
\frac{\mathrm{Q}}{\mathrm{Q}_{0}}-1=\lambda\left(\frac{\mathrm{N}}{\mathrm{N}_{0}}-1\right)
$$

$\mathrm{N}$ désignant soit le facteur nival seul considéré en l'occurrence, soit, par intégration de certains facteurs secondaires, une moyenne pondérée des différents facteurs intervenant dans le phénomène, $y$ compris le facteur nival naturellement.

Une deuxième remarque est la suivante :

M. Church, notamment dans le mémoire analysé ici, chiffre la qualité d'une corrélation ou covariation ( $\leftrightarrow H a r-$ mony ») par le pourcentage des écarts de prévision (écart $=\mathrm{Q} / \mathrm{Q}_{0}-\mathrm{N} / \mathrm{N}_{0}$ ) inférieurs ou égaux à $15 \%$ (de la valeur moyenne du facteur étudié). Nous désignons ce pourcentage par exemple par $R^{\prime}$.

Une telle notion conventionnelle nous semble impropre, car elle ne tient pas compte de la variabilité intrinsèque dudit facteur. $R^{\prime}$ peut être égal à 100 , et la corrélation proprement dite très faible et même inexistante.

Prévoir avec une erreur $\leqslant 15 \%$ un facteur qui, intrinsèquement, c'est-à-dire indépendamment des facteurs qui le conditionnent, ne s'écarte pas de plus de $15 \%$ de sat valeur moyenne, cela ne constitue pas une prévision.

A la notion « d'Harmony » utilisée par M. Ghurch, il est préférable de substituer la notion de coefficient de corrélation classique, qui s'exprime par l'une des deux formules suivantes :

$$
\begin{aligned}
\mathrm{R} & =\sqrt{1-\sigma^{2 / \mathrm{S}^{2}}} \text { (PEARson) } \\
\text { ou } \mathrm{R} & =\frac{x y}{\sqrt{2 x^{2} 2 y^{2}}} \text { (Bravais) (1) }
\end{aligned}
$$

1. Nous avons préconlisé également le coefficient $r=1-(0 / \mathrm{S})$ quí est l'expression d'un rendenent. Entre $R$ et $\vec{r}$ on a la relation $\mathrm{R}=\sqrt[2]{1-(1-r)^{2}}$.

\begin{tabular}{|c|c|c|c|c|}
\hline \multicolumn{2}{|c|}{ ENTRE : } & SAISON & \begin{tabular}{|l} 
HARMONY \\
$R^{\prime}(1)$ \\
Voir renvoi (1) \\
tableatu $n^{\circ} 3$
\end{tabular} & $\begin{array}{l}\text { Autruns } \\
\text { ET Fonmeles }\end{array}$ \\
\hline \multicolumn{2}{|c|}{\begin{tabular}{l|l}
3 stations : la Moselle & et le Main \\
Saint-Michel $(370 \mathrm{~m})$ & station : \\
Ramonchamp $(474 \mathrm{~m})$ & Jac d'Alfeld $(620 \mathrm{~m})$
\end{tabular} \mid} & $\begin{array}{l}\text { Octobre-mars } \\
\text { Avril-septembre } \\
\text { Année }\end{array}$ & $\begin{array}{l}0,73 \\
0,77 \\
0,91\end{array}$ & Dr. СнuRch. \\
\hline \multicolumn{2}{|c|}{3 stations $\quad$ le Neckar $\left\{\begin{array}{l}\text { el le Main } \\
1 \text { station : } \\
\text { Johanniskreuz (?) }\end{array}\right.$} & $\begin{array}{l}\text { Octobre-mars } \\
\text { Avril-septembre } \\
\text { Année }\end{array}$ & $\begin{array}{l}0,86 \\
0,64 \\
0,82\end{array}$ & Dr. Снurch. \\
\hline Lucerne & $\begin{array}{l}\text { èt moyenne de } 6 \text { stations } \\
\text { Berne }(572 \mathrm{~m}) \\
\text { Lucerne }(453 \mathrm{~m}) \\
\text { Glarius }(480 \mathrm{~m}) \\
\text { Coire }(610 \mathrm{~m}) \\
\text { Eigergletscher }(2.323 \mathrm{~m}) \\
\text { Safienplatz }(1.270 \mathrm{~m})\end{array}$ & $\begin{array}{l}\text { Période } \\
1925-1948\end{array}$ & $\begin{array}{l}\text { CoEFFICIENT } \\
\text { de } \\
\text { corrélation } \\
\mathrm{R}=0,965\end{array}$ & A. CoUtagne. \\
\hline \multicolumn{5}{|c|}{ COVARIATION THERMO-PLUVIALE } \\
\hline \multicolumn{3}{|c|}{ ENTre: } & $\mathrm{R}$ & \multirow[b]{2}{*}{$\begin{array}{l}\text { A. Covtagne. } \\
\left(\mathrm{H} / \mathrm{H}_{0}\right)-1=0,04\left(\mathrm{~T}-\mathrm{T}_{0}\right) \\
\text { Voir fig. } 7 \text {, page } 134: \\
\text { La Honille Blanche, } \\
\text { No spécial A, 1952, }\end{array}$} \\
\hline $\begin{array}{c}\text { Température à Strasbourg: } \\
\text { 1891-1934 } \\
\text { Pour l'année moyenne et } \\
\text { le mois moyen. }\end{array}$ & \begin{tabular}{|} 
Moyenne H des précipitation \\
Bâle (227 m) \\
Saint-Gall $(678 \mathrm{~m})$ \\
Zurich $(493 \mathrm{~m})$ \\
Lucerne $(497 \mathrm{~m})$ \\
Neuchatel $(487 \mathrm{~m})$ \\
Berne $(572 \mathrm{~m})$ \\
La Chaux-de-Fond $(989$ \\
Chur $(633 \mathrm{~m})$ \\
Engelberg $(1.560 \mathrm{~m})$ \\
Davos (1.017 $\mathrm{m})$ \\
$(1864-1900)$
\end{tabular} & 1s à 10 stations : & 0,97 & \\
\hline
\end{tabular}

BASSIN DU RHIN - TABLEAU $\mathrm{N}^{\circ} 2$

COVARIATIONS PLUVIALES (PRÉCIPITATIONS) 
BASSIN DU RHIN - TABLEAU No 3

COVARIATIONS FLUVIALES (DËBITS)

\begin{tabular}{|c|c|c|c|}
\hline ENTRE LES DÉBITS & Saison & $\begin{array}{c}\text { HAMMONY }^{\prime}(1) \\
R^{\prime}(1)\end{array}$ & $\begin{array}{l}\text { Avteuns } \\
\text { ET Formules }\end{array}$ \\
\hline $\begin{array}{c}\text { Du Neckar et du Main } \\
\text { Période : } 1925-1947\end{array}$ & $\begin{array}{l}\text { Octobre-mars } \\
\text { Avail-septembre } \\
\text { Année }\end{array}$ & $\begin{array}{l}0,52 \\
0,35 \\
0,48\end{array}$ & D). Снитск. \\
\hline ENTRE IES HYDRAULIGTÉS & PÉnLODE & $\begin{array}{l}\text { Confrichen } \\
\text { de } \\
\text { courclation } \\
\quad R\end{array}$ & A. Coutagne. \\
\hline $\begin{array}{c}\text { Du Rhin à Rheinfelden } \\
Q / Q_{0}\end{array}\left\{\begin{array}{l}\text { et de la moyenne : } \\
\text { Rhin superieur, Aar, } \\
\text { Linth, Reuss. } \\
Q^{\prime} / Q_{0}^{\prime}\end{array}\right.$ & $1927-1950$ & 0,94 & $Q / Q_{0}=Q^{\prime} / Q_{0}^{\prime}$ \\
\hline ENTRE LES DËBITS & PÉRIODE & & \\
\hline 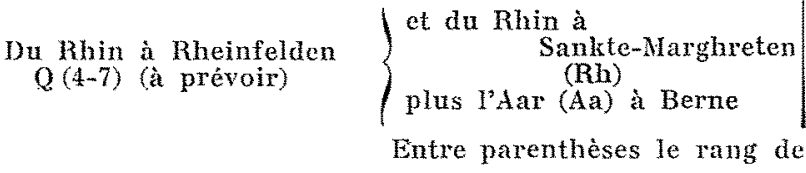 & $\begin{array}{l}1927-1947 \\
\text { Avril-juillet } \\
\text { mois : } Q(4-7)\end{array}$ & 0,645 & $\begin{array}{l}\text { Dr. Hoves. } \\
\qquad \begin{array}{l}(4-7)=0,390 \mathrm{Rh} \\
+0,612 \mathrm{Aa}-1,1\end{array}\end{array}$ \\
\hline
\end{tabular}

(1) Pourcentage des écarts de prévision inférieurs ou égaux a $15 \%$ de la valeur moyenne du facteur étudié.

TABLEAU $\mathrm{N}^{\circ} 4$ : RHIN

Corrélations A. Coutagis: : $\frac{\mathrm{Q}}{\mathrm{Q}_{0}}-1=\lambda\left(\frac{\mathrm{H}}{\mathrm{H}_{0}}-1\right)+y\left(\frac{\mathrm{X}}{\mathrm{X}_{41}}-1\right)$

\begin{tabular}{|c|c|c|c|c|c|c|c|c|}
\hline \multicolumn{3}{|c|}{ PHECIPTATIONS } & \multicolumn{3}{|c|}{ AUTTES FACTEURS } & \multicolumn{3}{|c|}{ DÉBT RECHEหChé : COEF } \\
\hline Saison & $\mathrm{H}_{0} \mathrm{~mm}$ & $\lambda$ & Paramètre et saison & $x_{0}$ & $\mu$ & SAISON & $Q$ & $\mathrm{R}$ \\
\hline
\end{tabular}

Station : Bare; Bassin versant en $\mathrm{km}^{2}: 35.925 ;$ Périolle : 1826-1925

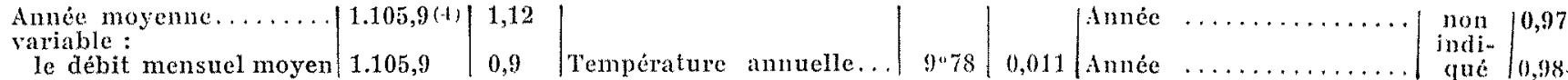

Station : Strashouro; Bassin versant en lin2 : $39.600 ;$ l'ériode : 1925-1948

\begin{tabular}{|c|c|c|c|c|c|c|c|c|}
\hline $\begin{array}{l}\text { Année hydrologique: } \\
\text { 1er octobre-30 sept. . }\end{array}$ & $1.240(3)$ & 1,125 & & & & $\mid \begin{array}{c}\text { Anné hydrologique: } \\
1^{\text {er }} \text { oclobrem } 80 \text { sept }\end{array}$ & 1088 & 689 \\
\hline $1^{\text {er }}$ octobre -30 sept. & $1.240(4)$ & 1,15 & Débit sept. précédent... & 1.097 & 0,17 & $1^{\text {er }}$ octobre-30 sept. & 1.085 & 0,94 \\
\hline Hiver & $474\{3\}$ & 0,48 & Précipit. été ... & & 0,52 & $1^{\text {er }}$ octobre-30 sept. & 1.085 & 0,985 \\
\hline Hiver & $474(3)$ & 0,9 & Débit sept. ...... & 1.093 & 0,34 & Hiver & 857 & $0,93(2)$ \\
\hline té & $766(3)$ & 0,9 & Précipit. hiver précédent. & $474(3)$ & 0,28 & Eté & 1.314 & 0,82 \\
\hline
\end{tabular}

(1) 0,943 , en ajoutant à la formule : $0,18\left(q / q_{0}-1\right), q=$ débit septembre et en prenant $\lambda=0,52$ et $\mu=0,54$.

(2) 0,96 , en considérant les écarts prohables au lieu des écartis moyens.

(3) 6 stations pluviométriques suisses (voir tableau $n^{\circ} 2$ ef pour les cortations annuclles, graphiciue 1)

(4) 10 stations pluviométriques suisses (voir tableau $\mathrm{n}^{\circ} 2$ et la Honille Blanche, $\mathrm{N}^{\prime 2}$ spécial A. 1952. p. 134, (lg. 7). 
qui sont, numériquement, équivalentes. Dans la première : $\sigma$ et $S$ représentent les écarts probables de probabilité et de corrélation. Dans la deuxième, $x$ et $y$ les écarts individuels $\left[\left(\mathrm{Q} / \mathrm{Q}_{0}\right)-1\right]$ et $\left[\left(\mathrm{N} / \mathrm{N}_{0}\right)-1\right]$ suivant nos notations ci-dessus.

Les corrélations pluvio-fluviales du Dr Church, relatives au Rhin à Rheinfelden sont assez faibles ( $\mathrm{Har}$ mony » $R^{\prime}=0,54$ entre $Q$ estival et $H$ hivernal; 0,75 entre $Q$ estival et couverture nivale; 0,90 entre $Q$ estival et $\mathrm{H}$ estival); celles que le Dr Chunch a tirées de l'étude du Dr. Hosck sont, en général, un peu meilleures : ¿ Harmony 》 $=0,62$ pour le Rhin à Rheinfelden; 0,90 pour le Rhin à Sankte-Marghreten, la Linth a WeesenBiäsche, la Reuss à Lucerne et l'Aar à Berne).

\section{DEUXIÈME PARTIE}

TabLEAUX ET Graphique

Enfin, nous avons groupe :

- Dans les tableaux Nos 2 et 3 , diverses covariations établies par le Dr Chunch, Ie Dr Hoscr et M. Cou. Tacise: la plus remarquable de ces études montre une liaison étroite entre les précipitations à Lucerne et la pluviosité en Suisse (noyenne des six stations précitées);

- Dans le tableau $N^{\circ} 4$, les corrélations établies par M. Coutagne à partir des données statistiques du Dr Chunch et des données fluviales de M. Portrat, Ingénieur en Chef des Ponts et Chaussées à Strasbours 1 : la meilleure de ces corrélations, relative au Rhin à Strasbourg (1925 à 1948) fait l'objet $d u$ graphique ci-après.

M. Coutagne indique, in fine, que la répartition mensuelle du déficit d'écoulement du bassin du. Rhin suisse, évalué annuellement à $500 \mathrm{~mm}$, au prorata de certaines fractions évaporométriques, conduit à une amplitude de la variation de la rétention totale du bassin égale à $160 \mathrm{~mm}$ (maximum fin avril). Un tel chiffre - donné sous toutes réserves - tendrait à témoigner en faveur d'une relativement faible influence de la rétention uivale, dans le conditionnement des débits d'été du Rhin suisse.

1. Yoil : La Houlle Blanche, n⿳ A-1952, pp. 130 à 134.
LE RHIN A STRASBOLRG (39.600 $\mathrm{km} 2)$ $1925-26$ à $1947-48$

ANNÉE HYoRologique (1 ${ }^{\text {er }}$ octobre-30 septembre)

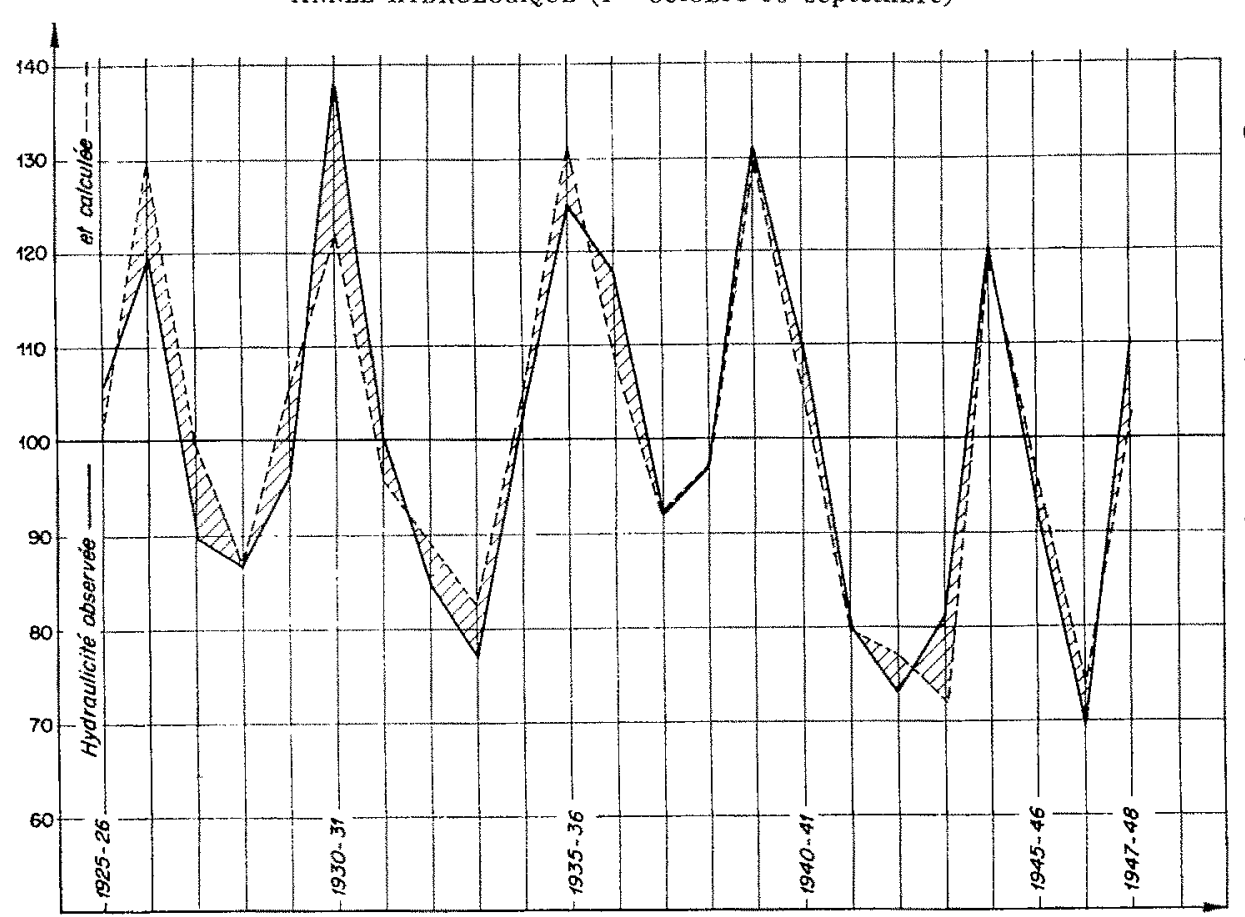

Corrélation entre le débit annuel, les précipitutions annuelles correspondautes et le débit du mois de septembre précédent $\mathrm{q}$ :

$\mathrm{Q} / \mathrm{Q}_{0}-1=1,15\left[\left(\mathrm{H} / \mathrm{H}_{0}-1\right)\right]$ $+0,17\left[\left(q / q_{0}-1\right)\right]$

$$
r=0,664 \quad \mathrm{R}=0,94
$$

écart moyen de probabilité : écart moyen de corrélation : $15,6 \%$ $5,20 \%$

$\mathrm{Q}_{0}=1.085 \mathrm{~m}^{3}$

$\mathrm{H}_{0}=1.240 \mathrm{~m}^{3}$

$q_{0}=1.097 \mathrm{~m}^{3}$

$\mathrm{Q}_{0}=1.040$ pour $1881-1950$

(à majorer de $10 \%$ pour avoir les précip. effectives du bassin).

Précipitations $=$ moy. arithmétique de 6 stations suisses : Berne, Lueerne, Glarius, Coire, Eigergletscher, Safienplatz.

\section{TROISIEME PARTIE}

\section{L'ANALYse de L'Étude dU Dl' HoEck}

La prévision du débit estival de quelques rivière suisses par la méthode de corrẻlation $\dot{a}$ plusieurs variables.

Nous renvoyons au résumé figurant en tête de cette élude dans le tome III des Rapports et Comptes rendus de la Commission des Faux de Surface, de l'Assemblée
Générale de Bruxelles, 1951, de lU,G.G.I., page 262, et nous citons les conelusions de M. Coutagne :

$1^{\circ}$ Les corrélations sont, dans l'ensemble, assez faibles. Elles sont, évidemment, troublées par les précipim tations estivales, et accessoirement par la fusion glaciaire (pour l'Aar, le bassin le plus glaciaire, R est relativement plus bas qu'ailleurs. A noter d'ailleurs la faible variabilité intrinsèque du débit à prévoir;

$2^{n}$ Les previsions, hasees sur la considération des fac- 
teurs météorologicues et hydrologiques antéricurs, sont aussi bonnes, sinon plus, que celles faisant intervenir le facteur nival, mesuré an sol (1);

$3^{\circ}$ La prévision à Rheinfelden est pratiquement de faible utilité. A noter que le facteur à prévoir est par lui-mème relativement peu variable, ce qui rend les prévisions peu utiles, en géméral, et contribue à diminuer le coefficient de corrélation. (Variabilité du debit moyen estival, chiffée par l'écart moyen $=9,8$ ot ;

4. Les méthodes mises en cuvre sont absolument identiques à celles que nous avons utilisẻes et préconisées dans toutes nos études antérieures. Voir notamment les tableaux, publiés dans la nowille Blanche, année $1952, \mathrm{n}^{\circ} \mathrm{A}$, concernant une vingtaine de bassins des Etats-Unis.) Un nouvel exemple relatif au Skagit River (Washington) - 10101904 - conduit à la formule :

$$
\begin{aligned}
& \mathrm{Q} / \mathrm{Q}_{0}-1=0,76\left[\left(\mathrm{~N} / \mathrm{N}_{0}\right)-1\right]+0,10\left[\left(q^{\prime} / q_{0}\right)-1\right] \\
& \text { et au coefficient } \mathrm{R}=0,94 .
\end{aligned}
$$

Le facteur nival $H=$ précipitations hivernales moins le débit hivernal, le facteur $q^{\prime}=$ debit antérieur au mois de novembre.

\section{Discussion \\ (President: M. ALEEHET)}

M. le Président remercic M. Corragne de nous avoin adressé son remarquable travail sur les mémoires de M. Churca et de M. Horek.

M. PARDí exprime son admiration pour M. Covtagne qui, malgré sa santé momentanémeni défieiente, s'allaque, avee un conrage inlassable et un esprit erilique avisé, à l'analyse de fous les mémolres qui lai sonl communiqués.

M. Pardé ajoute que, d'après une correspondance qu'il a eue avee $M$. Coutane, les prévisions dans lesquelles s'est lancé M. Church comportent une marge d'incertitude, parce que les débits d'origine nivale sur lesquels porte l'étude ne représentent qu'une fraction assez modique de l'écoulement total annuel. Sur le Rhin inférieur, même à Bâle, les grosses pluies de l'été peuvent tronbler les pronostics. Ceux-ci deviennent bien moins aléatoires, comme de juste, sur les hauts tronçons alpestres du réseau.

La séance est levée à 17 h. 40 .

\section{SÉANCE DU JEUDI MATIN 19 MARS 1953}

COMMUNICATTON DE M. LUXO

Ingénieur à Electricité et Gaz d'Algérie

\section{JAUGEAGES DE TORRENTS : PERCHE POUR LA MESURE DES VITESSES DANS LES OUEDS ALGÉRIENS (PRÉCISION ET CONDITIONS D'EMPLOI)}

Dans les oueds algériens, les relations hauteurs d'ean-débits dans les contròles naturels ou artificiels doivent ètre fréquemment vérifiées, du fait des modifcations fréquentes des abords des contrôles.

Les stations de jaugeage sont souvenl d'accès difficile et leur équipement ne peut être complet, les observatcurs qualifiés sont d'un recrutement difficile. Il est done nécessaire d'utiliser des instruments de mesure très simples et d'emploi aussi facile que possible.

L'instrument étudié est une perche en bois traité, le

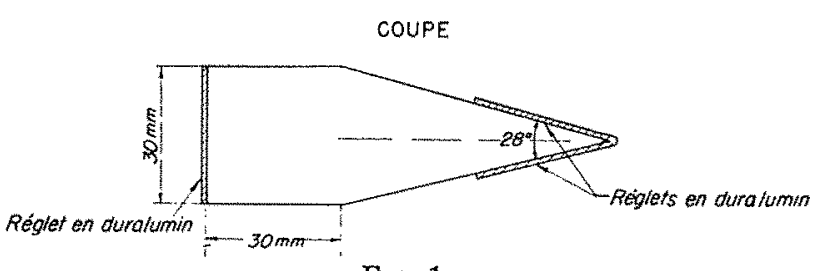

Fia. 1

profil est donné figure 1. La perche a $1,30 \mathrm{~m}$ de hauteur, le bas de la perche est proféźce par une plaque de dural et porte un petit ergot cylindrique de $1 \mathrm{~cm}$ de hauteur. De chaque côté de l'angle et sur la face opposée on dispose des réglets gradués en millimètres, le zéro de la graduation étant la face inférieure du petit ergot cylindrique (fig. 2).

1. C'est auss' l'avis des Suédois qui ont compnré les flewx méthodes dans leur pays. La méthode méteorologique est de plus d'une application plus simple et moins oncrease et permet d'utiliser des statistiques a plus longue durée.

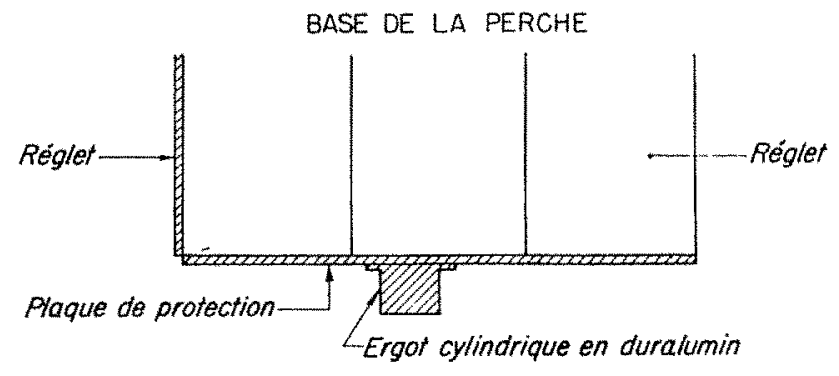

Fix. 2.

L'utilisation est la suivante:

On lit d'abord la hauteur d'eau $h_{*}$ en presentant au courant langle de $28^{\prime \prime}$, puis on fail tourner la perche su" son ergot en prenant soin qu'elle ne glisse pas et on lit la hauteur de remonle $h_{t}$ du remous sur la face plane perpendiculaire au courant. La différence des lectures $\Delta h$ est :

$$
\begin{aligned}
\Delta h=h_{s}-h_{i} & =h+(\alpha \mathrm{V} 2 / 2 g)-h \\
\Delta h & =a \mathrm{~V}^{2} / 2 g
\end{aligned}
$$

$V$ étant la vilesse moyenne sur la verticale de mesure:

$$
\mathrm{V}=\frac{1}{\sqrt{\alpha}} \sqrt{2 g \Delta h}=k \sqrt{2 g \Delta h}
$$

Nous avons vériflé que la constante $k$ est très voisine de l'unité.

L'etalonnage de la perche a éte fait: dans un canal trapézoïdal (au Laboratoire Neyrpic d'Alger) $(B=1,20 \mathrm{~m}$; $b=0,40 \mathrm{~m} ; h=0,64 \mathrm{~m})$. Le débit était mesuré au moyen d'un déversoir Rehbock.

On remarque que la perche surestime le débit de 2 a $10 \%$ dans ces conditions d'emploi.

Nous avons par ailleurs comparé les mesures de débits effectués in situ au motlinet et a la perche. La perche surestime le debit de 1 a $5 \%$ par rapport au moulinet.

Les mesures sont d'autant plus sensibles que la vitesse est plus grande. 
L'emploi de la perche est évidemment limité aux profondeurs inférienres à $0,60 \mathrm{~m}$ et aux vitesses inférieures ì $2,50 \mathrm{~m} / \mathrm{s}$. Nous avous pu. effectuer des mesures jusqu'à $12 \mathrm{~m}^{3} / \mathrm{s}$ avec des vitesses supérieures à $2 \mathrm{~m} / \mathrm{s}$ (sur l'Oued Djendjen inférieur).

La perche peut permettre de mesurer des débits par de faibles profondeurs d'eau lorsque le moulinet est inutilisable.

On peut choisir la section de mesure sur une barre rocheuse, par exemple, et c'est un avantage de l'emploi de la perche de ne nécessiler aucun équipement de la section (passerelle, treuil).

Son interêt principal est la simplicité et la rapidité de la mesure, les observateurs ont deux hauteurs d'eau à mesurer par verticale, il suffit qu'ils sachent lire l'échelle ef écrire les chiffres sur des fiches préparées à l'avance.

Une perche tout à fait analogue a été étudiée par H. G. WiLM et H. C. Stoney (Velocity Head Rod Calibraled for Measuring Streamflow. Civil Eng., Vol. 14, pp. 475$476,1944)$.

\section{Discussion}

(Président : M. HupNer)

M. RẺMÉñÉnas indique que l'intérêt de cet appareil est surtout d'être robuste et facilement transportable : une amélioration possible consisterait à encastrer dans la perche un tube de pitot classique: la hauteur $\mathrm{K}$ ( $2 / 2 g$ ) Jue sur le Pitot serait plus précise que celle du panache, qui doit être très fluctuante et difficile à evaluer par lecture directe 1.

M* le Président remarque que c'est surtout un instrument de prospection.

M. Boyen rappelle que la perche peut permettre de mesurer des débits lorsque le moulinet est inutilisable, soit par de faibles profondeurs d'eau, soit par suite des difficultés d'installation d'une section de jaugeage.
Répondant à M. Varler, M. Boysn indique que la vitesse mesurée est plutôt la vitesse superficielle qui, dans les oueds algériens à faible profondeur doit donner une idée assez approchée de la vitesse moyenne.

M. Le Président suggère au Service des Etudes el Recherches Hydrauliques d'E.D.F. d'essayer une perche empruntée à E.G.A. dans certaines sections de la Durance it très faible profondeur d'eau.

M. VARLET rappelle qu'on peut aussi estimer les vitesses par les vibrations d'uno canne plongée dans l'eau.

M. Ie Président remercie M. Luxo et souhaite que l'on fasse des essais de cet instrument de prospection sur les rivières françaises.

COMMUNICATION DE MM. LABAYE ET DURANTON

\section{UTILISATION D'UNE GALERIE SOUTERRAINE EN RÉSERVOIR D'ÉCLUSÉE}

Le mémoire in extenso de MM. Labaye et DuRanton el sa discussion sont publiés pages 736 et suivantes du présent numçro.

COMMUNICATION DE M. FAURE

\section{CALCUL DES PERTES D'ÉNERGIE DANS UN ESTUAIRE A MARÉE : PRINCIPE ET EXÉCUTION} AU MOYEN DUNE MACHINE MATHËMATIQUE

Le mémoire in extenso de M. Faure et sa discussion sont publiés pages 748 et suivantes du présent numéro.

\section{SÉANCE DU JEUDI APRÈS-MIDI 19 MARS 1953}

La séance est ouverte à 14 h. 30 , sous la présidence de M. Gamiel.

\section{NOUVELLES MÉTHODES DE CALCUL PRATIQUE DES ECOULEMENTS DE FILTRATION NON PERMANENTS A SURFACE LIBRE}

EXPOSĚ THĚonIQUE de M. SchNeEBELI

Mise au polnt des calculs analogigues par M. HUARD de la MARre

En raison de leur affinité, les deux communications

1. Dans une lettre postêrieure à la sêance à laquelle il n'assistait pas, $M$. Luxo objecte qu'll faudrait rendre mobile le Pitot a prise de pression totale t, de plus, faire plusicurs lectures par verticale, ce qui complique le procédé et augmente le temps de mesure, et que la hauteur lue sur un Pitot placé dans un oned torrentiel est également très fluctuante. ci-dessus ont été discutées ensemble, après l'exposé de M. HUaRd DE LA MARRE.

Les mémoires in extenso et leur discussion sont publiés pages 761 et suivantes du présent numéro.

\section{COMMUNICATION DE M. RÉMËNIÉRAS}

\section{ESSAI SEMI-INDUSTRIEL DES DRAINAGES ÉLECTROOSMOTIQUES D'UN TALUS}

Le mémoire in extenso de M. RGméniénas et sa discussion sont publiés pages 777 et suivantes du présent numéro. 


\section{Session des 17, 18 et 19 juin 1953}

Ont participé aux travaux de cette session :

a) Conseil d'Administration :

M. le Président Masst.

MM. Gariel, Aubert, Chevrier, Coyne, Fenrender, Hupner, Mary, Nyzery, de Rovville.

b) Adhérents :

L'Electricité de France, représentée par MM. Baucheron, ne Beaureqard, Binet, Birard, Cappus, Destenay, Duclos, Dumaine, Dupux, Kammerlocher, Larriev, LÉonino, Letanche, Longuemare, Lugiez, Marquenet, Molbert, Obolenski, Piguet, Ravier, Rodrer, TruviDIC, VANTROYS, VassiviḱRE.

La Société Hydroélectrique des Pyrénées, représentée par M. Henvế

Le Service des Ponts et Chaussées de la Loire-Maritime, représenté par MM. Siegrried et Ballade.

Les Etablissements Neyrpic, représentés par MM. Almeras, Comen de Lara, Condolios, Lafaix, Le Ménestriel, R. MEYER.

La Société Lyonnaise des Eaux, représentée par M. Lénr.

La Société des Forges et Ateliers du Creusot, représentéc par M. Sochat.

Le Bureau d'Etudes A. Coyne et J. Bellier, représenté par M. Legros.

L'Entreprise Ossude, représentée par M. Valloant.

La Société Générale d'Entreprises, représentée par M. Connet

La Société de Construction des Batignolles, représentéc par M. Vieuxtemps.

M. Dalapd.

\section{c) Comité Technique :}

M. le Président Barrillon.

M. Darrates, Président de la Commission pour l'Etude des PD?.
M. Escande, Président de la Commission de Révision du Code d'Essais des Installations Hydrauliques.

M. Messines du Sourbien, Président de la Sous-Scetion de Glaciologie.

M. Serra, Président de la Commission du Déficit d'Ecoulement.

M. Brengeron, Vice-Président de la Section « Machines».

M. RÉméniťras, Secrétaíre Général.

MM. Altard, Arlinx, Bablon, Banal, Bourgulgnon, Bouvard, Chabert, Chamayou, Conescent, Danel, Durand R, Esclangon, Ferrindon, Germain, Gubat, Gougenheim, Guidel, Habgelen, Labaye, Langlois, Larrieu, Ledoux, Legendre, Lescall, Mattre, Monlat, Parod, Sauvage de Saint-Marc, Suquet, Thimel, Valdembois, VENNIN.

MM. Luaiez et Dumaine, représentant M. Ferny.

M. TuRc, représentant M. HÉniN.

M. Devimeux, représentant M. J. Launent.

\section{d) Invités :}

M. Volken, Ingénieur Principal des Travaux du Zuyderzée (Hollande).

M. Cupenus, Ingénieur au Waterloopkundig Iaboratorium de Delft (Hollande).

M. Binot, Professeur à la Faculté des Lettres de Lille.

M. Nombert-Casteret, spéléologue.

MM. Archambault, Bafour, Ch. Beav, Bournuen, Futchy, lisyy, Léprevex, Piver, Putatei, Revol, Siguner, Veiver.

\section{S'étaient excusés :}

Mlle Garenc, MM. Coutane, Coutratu, Duffaut, Fenny, Frolow, Giguet, Hardy, Lacombe, Merier, Mrssenand, Montagne, Olvien-Martin, Oudn, Pfahl, Schlad, SenTENac, Tissier, Tnicart, Vinent, E.D.F., Region d'Equipement Hydraulique Garonne a Toulouse, E.D.F, Région d'Equipement Hydraulique Alpes III a Marseille.

\section{SÉANCE DU MERCREDI APRÈS-MIDI 17 JUIN 1953}

La session est ouverte sous la présidence de M. BARrullon, Président du Comité Technique.

\section{VIE DE LA SOCIÉTÉ}

\section{Approbation du procès-verbal de la session des 18 et 19 mars 1953}

Le procès-verbal de la session des 18 et 19 mars 1953 , déposé sur le bureau, n'ayant donné lieu à aucune observation, est adopté ipso facto.

\section{Admissions au Comité technique}

Sont admis au Comité Technique :

M. Trson, Professeur à l'Université de Gand (Belgique).

M. Lamounoux, Ingénieur en Chef des Ponts et Chaussées, Chef de la $1^{\text {se }}$ Circonscription Electrique, Paris.

M. Praki, Ingénieur en Chef des Ponts et Chaussées, Chef de la $4^{\mathrm{e}}$ Circonscription Electrique, Limoges.
M. Pirault, Ingénieur en Chef des Ponts et Chaussées, Chef de la $3^{\circ}$ Circonscription Electrique, Nantes.

M. Marquener, Ingénieur au Laboraloire Nalional d'Hydraulique, Chaton.

M. Bonnin, Ingénieur au Service des Etudes et Recherches Hydrauliques d'Electricité de France.

M. Schneebela, Ingénieur au Service des Eludes et Roeherehes Hydrauliques d'Electricite de France.

M. Cappes, Ingénieur an Service des Etudes et Recherches Hydrauliques d'Electricité de France.

M. Almeras, Ingénieur aux Fts Neyrpic.

M. Boudan, Ingénieur au Iaboratoire Dauphinois dHydraulique, Grenoble.

M. J.-P. Rayxaud, Ingénieur au Laboraloire Dauphinois d'Hydraulique, Grenoble.

Ces nominations ont été ratifices par le Conseil d'Administration le 24 juin 1953 


\section{Documents reçus :}

Oubrages francais :

- lompes ventiluleurs compressenrs, par A. DE KowäTs et G. Desmur.

- Annaleswemoires et notes techniques da service du Ganie Rural et de lHydraulique Agricole, fascicules $72 a-72 b$.

- Programme générul d'alimcntation en eau polable du lépartement du Puy-de-Dóme, par H.-F. PATrux.

- Equipement 1952, par Electricité de France.

- Pluviométrie, Bulletin de récapitulation 1952, par la Compagnie Genérale des Eaux (Exploitation de Nice et Annexes).

- Liste des travaux publiés de 1913 à 1953, par J. Kampe DE FEnivr.

- Elade des corrélations plavio-fluviales (régionales, périodiques el interpériodiques) du Wurtemberg (Extrait de la "Revue Générale de l'Hydraulique »), par A. Coutagne.

- Contribution à l'étude de la pénétration des eaux pluviales dans le sol, par A. Coutagne.

- Recueil des observations hydrometriques, année 19511952, par la Direction des Travaux Publics (Bureau de l'Inventaire des Ressources hydrauliques de Tunisie).

Onvrages étrangers :

- Eudes sur modèle réduit pour les mannes des aqueducs de l'éclase de la Sambre d Namir, par J. LAMOEN.

- Energies libanaises, par Innamm ABD-EL-AL, Directeur général des Travaux Publics et du Contrôle des Sociétés.

- Annuaires hydrologiques allemands pour le bassin supérieur du Rhin, le bassin inférieur de l'Elbe (année 1952), par le Landesstell für Gewässerkunde, Karlsruhe.

De l'American Geographical Society :

- Report on the Northern American glaciers, par P.D. Batnd.

- Glacier fuctuation in Northwestern North America within the past six centuries, par D. B. LAwrence.

- Glaciological Research in Alaska, par W. O. Field.

- Polen profles from Southeastern Alaska, par C.-J. Hevsser.

De l’Union Géodésique et Géophysique Internationale:

- Preliminary infiltration investigations in New Zealand. par D.J. Nonduye et D. A. Campuelt.

- Types of soil erosion prevalent in New Zealand, par D. A. Canphell.

- Soil erosion and its control in the South half of the North Island of New Zealand, par A.F. GReEnhali.

-. Soil erosion and its control in the Northern half of the South Island of New Zealand, par D.R. WILkts.

- Soil erosion and its control in the Southern half of the South Island of New Zealand, par H.C.H. Pearce.

- Soil erosion and its control in the Southern half of the South Island of New Zealand, par G. G. CaLden.

- Variability of flow in lake-controlled rivers of New Zealand, par Allan D. BExHaM.

- Diffculties in obtaining and presenting idrologic data, par C. SchNackendera.

- Lake IVaikaramoana, New Zealand, par N. R. Canten et B. Se. Amvzle.

- Anales sismologicos, 1948-49-50 (Obscrvatorio Central, Buenos Aires).

- Resumen $y$ analisis de Observaciones de Flertricidad Atmosferica (Pilar), années 1924-1936. Servicio Meteorologico Nacional, République Argentine.
OtzTalen Ache, Oesterteichischer Wrasserkraft-hataster (Studienausschuss des Oesterreichischen Wasserwirtschattverbandes, Wien).

M. Le Prósident signale aussi une page consacrée à M. De hovvine, Administrateur de la S.H.F, Président de la Section d'Hydraulique Fluviale et Maritime dans la Technique Moderne Construction cuméro de mai $1953)$.

\section{Revision du Code d'Essais}

Lat Commission de Révision du Code d'Essais des Inslallations Hydrauliques, réunic le 24 mars sous la présidence de $M$. Fiscande, a continué l'examen du fascicule 6 par les chapitres spéciaux aux pompes, proposés par les représentants des constructeurs.

Le chapitre «Mesure des débits par déversoirs 》 (fascicule $\mathrm{n}^{\circ}$ 5) sera communiqué aux membres de la Commission dans sa forme définitive avant impression.

Des projets de textes relatifs aux mesures des débits par la méthode de l'écran d'Anderson, la méthode d'Allen et les appareils déprimogènes, ainsi qu'une deuxième lecture des caractéristiques de fonctionnement des machines seront proposés á la Commission à l'automme prochain on vue de l'édition des fascicules 5 et 6 ainsi complétés.

\section{Etude du déficit d'écoulement}

La Commission pour l'étude du déficit d'écoulement s"est réunie ce même jour 17 juin sous la présidence de M. SerRa avec l'ordre du jour suivant :

- Etude générale de l'inertie hydrologique d'un bassin. Courbes de décroissance du débit, mensuel on journalier, pendant les périodes à précipitations nulles ou faibles (cours d'eau intertropicaux, cours d'eau du Moyen Orient, etc.), par A. Coutagne.

-.. Calcul des drainages pour de courtes périodes, par M. TuRc.

- A propos du déficit d'écoulement du bassin de la Maronne, par A. Coutagne.

\section{Informations diverses}

Sont communiquẻes ou rappelées au Comité Technique:

- L'organisation de la « Tournée Glaciologique 》, annuelle, du 20 au 23 juillet 1953 , en Suisse (voir plus loin le compte rendu de cette Tournée).

- L'organisation des \& Troisièmes Journées de l'Hydraulique $\gg$ du 12 au 14 avril 1954 a Alger (voir plus loin le programme de ces «Journées»).

Les publications parues ou en preparation depuis la dernière session.

- L'organisation en automne 1954 du Colloque International sur les oseillateurs et servomécanismes non linéaires, par le Centre National d'Etudes des Telécommunications, 24, rue Bertrand, Paris $\left(7^{\circ}\right)$

\section{COMMTVICATION DE M. LEGENDRE}

\section{DISTRIBUTION DES VITESSES MOYENNES POUR L'ÉCOULEMENT TURBULENT DANS LES CONDUITES CYLINDRIQUES}

Le mémoire in extenso de M. Learsone et sa discussont publiés pages 793 et suivantes du présent numéro.

\section{COMMTVICATION DE M. YOLKER}

\section{LA MARÉE DE TEMPÊTE DU I TR FÉVRIER 1953 ET SES CONSÉQUENCES POUR LES PAYS-BAS}

Le mémoire in extenso de M. Vouken et sa discussion sont publiés pages 798 ef suivantes du présent numéro. 


\section{SEANCE DU JEUDI MATIN 18 JUIN 1953}

La séance est présidée par M. Baralloon, Président du Comité Technique.

\section{COMMUNICATION DE M. P. CAPPUS}

\section{TERRAIN EXPËRIMENTAL D'ALRANCE : CALCUL. DU BILAN HYDROLOGIQUE ÉTUDE DE L'ÉVAPORATION}

\section{Résumé}

Nous avons dabli le bilan hydrologique du terrain expérimental d'Alrance pour les années 1951 et 1952 .

Les pertes par évaporation $E$ sont données par l'équalion du bilan:

$$
\mathbf{P}=\mathrm{Q}+\mathbf{E}+\Delta \mathrm{R}
$$

P : pluie, $Q$ : débit, $\Delta \mathbf{R}$ : variation de la réserve. L.ES DONNEES (fig. 1)

- Les pluies sont données par 11 pluviomètres répartis sur les $3,7 \mathrm{~km}^{2}$ du bassin; les neiges pax la cuve à neige; les débits par la station à déversoir muaie d'un limnigraphe.

- Pour déterminer $\Delta R$, on pouvait utiliser :

- Les niveaux phréatiques, mesurés en 9 points du bassin. Mais ils ne donnent $\Delta \mathrm{R}$ quavec peu de précision : leur nombre est en effet insuffisant pour déterminer le niveau phréatique sur tout le bassin; d'autre part le nivean phréatique diffère du niveau de saturation de la hauteur de la frange capillaire, qui n'est pas connu exactement; enfin certains phénomènes capillaires rendent encore ces mesures plus incertaines.

Nous n'avons utilisé les niveaux phréatiques que pour déterminer la saturation totale du bassin, quand toutes les profondeurs phréatiques sont inférieures a la hauteur de la frange capillaire.

- La courbe de tarissement : la réserve souterraine est constituée par un grand nombre de nappes alimentant de petites sourees ou des zones de suintement. L'égalité à deux instants de la somme des débits de l'ensemble des nappes constitue donc une forle présomption en faveur de l'égalité de la

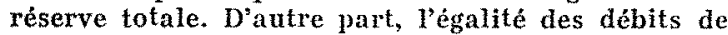
la rếserve à deux instants permet d'affirmer l'égalité des réserves si chacun des instants suit de peu une période de saturation complète : à partir de la saturation, le terrain ne peut en effet se vider que d'une seule façon.

Nous ayons comparé les pertes par evaporation calculées $\mathrm{E}=\mathrm{P}-\mathrm{Q}-\triangle \mathrm{R}$, aux données des évaporomètres Piche.

En effet, si l'évaporation à partir de la rondelle de papier buvard n'est pas la même que l'évaporation a partir du sol ou des plantes, l'évaporomètre Piche peut cependant donner les variations relatives du pouvoir évaporant de l'atmosphère. Malheureusement les évaporomètres utilisés sont à l'abri du rayonnement solaire direct et partiellement à l'abri du vent (abri à persiennes) et ces deux facteurs augmenteraient l'évaporation. Cependant nous avons vérifié que pour des périodes de l'ordre du mois, la moyenne de la vitesse du vent varie peu; ses variations auraient une influence négligeable sur l'évaporation Piche hors abri.

Ayant vérifié qu'à l'échelle mensuelle et même décadaire, l'évaporation Piche est proportionnelle a l'écar psychrométrique, nous avons pu reconstituer l'éraporation Piche des mois d'hiver d'après les enregistrements d'un thermomètre et d'un hygromètre.
Bilans annuels

\section{1: du 17 auril 1951 au 30 janvier 1952}

Chacune des dates limites est précédée d'une période de saturation (en mars et avril 1951, on ne mesurait pas encore les niveaux phréntiques, mais on peut affrmer la saturation à la fin du mois de mars, en raison des pluies antéricures importantes et du fort débit de la nappe (environ $250 \mathrm{l} / \mathrm{s}$ ) qu'on he retrouve à l'hiver 1951-1952 qu'en periode de saturation). D'autre part, le débit de lat

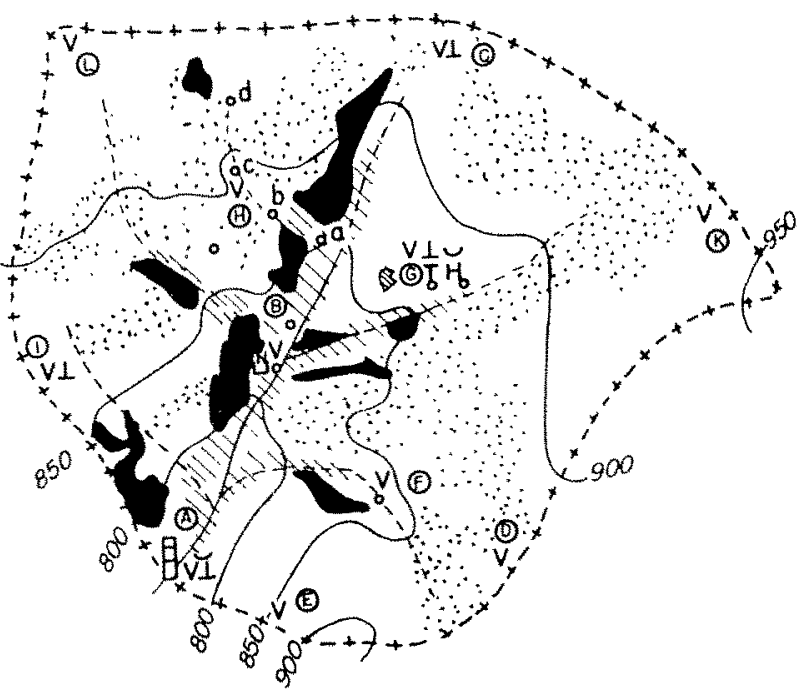

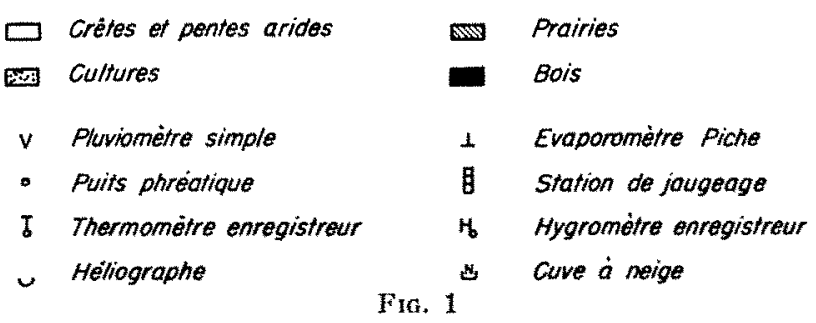

nappe est le même aux limites $(90 \mathrm{l} / \mathrm{s})$. On peuL donc affirmer l'égalité des réscrves aux deux dates limites.

$$
\begin{aligned}
& P=1.004 \quad Q=433 \quad \Delta R=0 \\
& \text { Pertes par evaporation. } \ldots=571 \\
& \text { Fvaporation Piche } \ldots \ldots \ldots=550
\end{aligned}
$$$$
\text { 1952: du 30 jampier an } 8 \text { decembre }
$$

Chacune des dates limites est précédée d'une périodo de saturation (profondeurs phréatiques inférieures a la hauteur capillaire) après laquelle le débit de la nappe decroit rapidement. Le débit de la nappe est le même aux deux limites $(90 \mathrm{l} / \mathrm{s})$; les profondeurs phritatiques ne different pas de plus de $15 \mathrm{~cm}$. On peut done affemer légalité des réserves aux deux dates limites.

$$
\begin{gathered}
P=813 \quad Q=368 \quad \Delta \mathrm{R}=0 \\
\text { Pertes par evaporation. } \ldots=445 \\
\text { Evaporation Piche } \ldots \ldots \ldots=760
\end{gathered}
$$

FTUDE DES PERTES PAR EVAPORATHON

1) Les bilans 1951 et 1952 montrent l'importance enorme de l'infiltration et de l'évaporation, of le peu d'importance du ruissellement (fig. 2).

2) IIs font apparaître une discordance entre les pertes par exaporation E et les totaux de l'evaporation Piche, qui ne sont pas dans le meme rapport. 


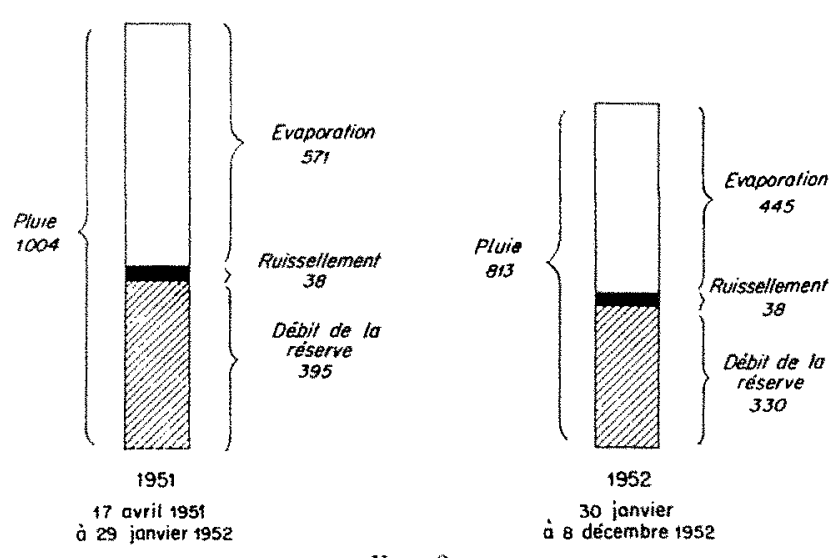

Fir. 2

lilans annuels.

Cette discordance peut-elle s'expliquer :

-. Par l'imprécision sur E? Non, car celte imprécision est au maximum de $\pm 70 \mathrm{~mm}$ pour chaque annce;

- Par un détarage des évaporomètres Piche entre 1951 et 1952? Non, car la correction du détarage que l'on aurait pu supposer augmenterait encore l'évaporation Piche en 1952;

- Par le fait que les évaporometres sont à l'abri du soleil? Non, car l'année 1952 a eté beaucoup plus ensoleille que l'amnée 1951.

Si les pertes par evaporation ne se sont pas accrues entre 1951 et 1952 proportionnellement au pouroir evaporant de l'atmosphère, e'est que la faible pluviosité du printemps et de l'été 1952 a entrainé un abaissement considérable de la nappe phréatique. Pendant une partie de l'été, la nappe etait trop basse pour alimenter la transpiration des plantes. L'évaporation n'était plus alimentée que par les pluies, qui précisément étaient faibles.

Le $1^{\text {er }}$ juillet 1952 , les profondeurs du niveau phréatique étaient :

en B, F, H: $1 \mathrm{~m} ; \quad a, b: 1,5 \mathrm{~m} ; \quad d: 1,6 \mathrm{~m} ; \quad c: 2 \mathrm{~m}$

Le bassin d'Alrance comprend:

$30 \%$ de pentes et de crêtes où la profondeur de terrain perméable ne dépasse pas $1 \mathrm{~m}$.

$30 \%$ de zones de cultures, sur le plateau, oủ l'épaisseur de terre dépasse souvent $2 \mathrm{~m}$. Mais les profondeurs c et $d$ montrent que la transpiration des cultures était impossible ( $h$ capillaire $+h$ de la zone d'aération $=$ environ 100 à $150 \mathrm{~mm}$ ). D'ailleurs une grande partie des cultures (céréales) arrive a maturité et commence à sécher en juillet.

30 \% de prairies, dans les vallées. Là aussi, les niveaux phréatiques montrent la difficulté (plus nette au $1^{\text {n }}$ août) d'alimenter l'évapotranspiration.

Lohservateur charge des mesures a effeclivement confirmé qu'en juillet-août 1952 le platcau était complètement desseché, les prairies de la vallée qui d'habitude conservent l'humidite plus longtemps souffaient également de la sécheresse. Au cours de cette période de sécheresse, les arbres seuls pouvaient aller chereher l'eau en profondeur et continuer ì transpirer, mais ils ne couvrent que $10 \%$ du bassin.

Pendant l'été 1951, par contre, le débit minimum de la nappe est le même que dans la première quinzaine de juin, ou dans la dernière semaine d'octobre 1952: le niveau de la nappe est done sensiblement le même pendant ces trois périodes : on vérifie en 1952 que ce niveau est suffisant pour permettre la transpiration. La transpim ration a donc été alimentée pendant toute l'année 1951 .

En tenant compte du fait qu'en 1951 les pertes par exapotranspiration sont du même ordre que l'évaporation Piche, on peut répartir les pertes en $1952(445 \mathrm{~mm})$ de la façon suivante :
- 13 juin an 14 seplembre : pouvoir évaporant de lalmosphère trés èlevé (P'iche : environ $395 \mathrm{~mm}$ ); nappe phrealique trop basse; l'évapotranspiration n'est alimenté que par les pluies (115 mm) et lex abbres (10) $\%$ de la surface : environ $40 \mathrm{~mm}$ de transpiration). Perle par exapolranspirntion: enriron $150 \mathrm{~mm}(Q$ : enviton $5 \mathrm{~mm})$.

- 15 seplembre au 8 décembre : pouvoir évaporant de latmosphère peu élevé (Piche : environ $80 \mathrm{~mm}$ ) et inféricul à $\mathrm{P}-\mathrm{Q}=396-115=281 \mathrm{~mm}$.

Perle par évapotranspiration : environ $80 \mathrm{~mm}$. Lexcedent de pluie $\mathrm{P}-\mathrm{Q}-\mathrm{E}=200 \mathrm{~mm}$ ramène la réserve de son minimum à son maximum.

- 30 janvier au 15 juin : pouvoir evaporant de l'atmosphère (Piche enviton $2 S 0 \mathrm{~mm}$ ) supérieur a $\mathrm{P}-\mathrm{O}$ $=\$ 02-247=55 \mathrm{~mm}$.

Porte par evapotranspiration : environ $215 \mathrm{~mm}$ (inférieure à l'évaporation Piche peutedre parce que les pentes et les erétes sont déjà inaptes a Tévapotranspiration à la fin de mat et at début de juin). La réserve foumit $\mathrm{F}-(\mathbf{P}-\mathbf{Q})$ : environ $160 \mathrm{~mm}$ à l'évapotranspiration et diminue de son maximum jusqu'au voisinage de son minimum.

\section{Conclusion}

1. Il faut noter l'importance primordiale de l'évaporation et de linfiltration el le peu d'importance du ruissellement.

2. L'vaporation Piche fournit un ordre de grandeur des pertes par évaporation (à véxifier sur d'autres hilans annuels et si possible sur des bllans mensuels) quand la nappe phréatique est assez peu profonde ou les pluies suffisantes pour alimenter. l'érapotranspiration.

$3^{\circ}$ Le pouvoir évaporant de l'atmosphère n'est qu'un tacteur de l'évapotranspiration.. Celle-ci dépend encore :

- du développement et de l'àge de la végétation;

- du niveau de la nappe et de l'état d'humidité du sol dans la zone des vecines (qui détermine la possibilité ou l'impossibilité de l'évapotranspiration).

Il est utile de mesurer avec précision les facteurs météorologiques de l'évapotranspiration. Mais il est beaucoup plus important :

- De faire quelques observations sur la végétation;

- Et surtout de déterminer l'aptitude de l'eau souterrame à alimenter l'évapotranspiration par l'étude de la nappe phréatique et les mesures d'humidié du sol.

\section{Discussion}

M. Ie Président remercie M. Cappus et souligne l'importance des observations qualitatives faites sur place, notamment sur la végetation. Il précise que Ia valeur de la réserve est une fonction univoque, non seulement du débit, mais de la vilesse de variation du débit. D'autre part, il suggère que lorsqu'on disposera de statistiques assez longues, le bilan soit étudiè à l'échelle mensuelle qui permet de mieux isoler les cas favorables à une comparaison des variations de déhit et notamment d'éliminer les périodes neigeuses qui bronillent les résultats.

M. Meyer signale que, d'après une étude de M. JaEger, il peut exister dans les écoulements en milieu poreux un régime analogue au régime torrentiel des écoulements en canaux: en régime torrentiel, le débit né dẻpend pas du niveau aval, il peut done $y$ avoir plusieurs débits différents pour un même niveau aval; comme dans les canaux le régime torrentiel est le régime qui s'établit normalement quand les pentes sont fortes, ce qui a l'air d'ètre le cas dans certaines parties du terrain d'expériencc d'Alrance.

M. la Président rappelle que la courbe de tarissement peut être absolument quelconque, puisqu'on peut ima- 
giner un milieu et une forme de terrain susceptibles de donner n'importe quelle forme de tarissement.

Répondant à une question de M. Lugrez sur la corrélation entre les résultats de l'évaporomètre Piche et les mesures failes au psychromètre, M. Cappus précise qu'il s'est borne a donner un graphique a titre d'exemple de cette corrélation, mais que M. Coutagne a établi statisliquement la formule:

$$
e=v(1+0,1 v)
$$

dans laquelle e représente l'évaporation Piche, $\vartheta$ l'écart psychrométrique et $V$ la vitesse du vent. L'exemple donné par M. Capros révèle entre les valeurs décndaires de e et $y$ un coefficient de proportionnalité de 1,5 correspondant ì la vitesse moyenne du vent qui est, à Alrance, de lordre de 4 ou 5 mètres par seconde. L'étude des valeurs joumalières de et $\theta$ révèle par rapport à la droite de régression des écarts qui montrent l'influence de la vitesse $d u$ vent mais ne permettent pas d'etablir une corrélation très sûre.

M. Luarzz pense qu'il pourrait être intéressant d'étudier la variation de la valeur en eau de la neige recouvrant le bassin par des mesures directes faites en nombre suffisant avant et après la crue due à la fusion.

M. Cappus estime cependant qu'à Alrance les faibles précipitations neigeuses sont gênantes pour un calcul du

M. Aruenx signale une méthode de calcul de l'éraporation en fonction du défieit de saturation et de la vilesse du vent. Cetle méthode due à Rosenan (Israël) se présente sous la forme d'un abaque dont la lecture serait plus simple que l'emploi de l'évaporomètre et plus sure que les formules de corrélation de l'évaporation avec l'écart psychrométrique et la vitesse du vent. bilan, mais insuffisantes pour une étude de la fusion.

COMMLNICATION DE M. ARLERY

\section{QUELQUES REMARQUES A PROPOS DE L'APPLICATION DES CONCEPTIONS DE C. W. THORNTHWAITE A UNE CLASSIFICATION DES CLIMATS DE LA FRANCE}

\section{Résumé}

L'evapotranspiration potentielle $\mathrm{F}$ des vegetax est celle qui serait rélisée si hea évaporable faisat l'objel d'un renouvellement continu : elle est uniquement liee aux propriétes immédintes de latmosphère. On peut la considérer comme une caractéristique du climat et la limite supérieure de l'évapotranspiration effective, ou réelle, qui est limitée par l'humidité du sol ou quantité d'eau susceptible de s'évaporer, et variable suivant les concepts de «transfert de vapeur».

L'évapotranspiration potentielle est définic par C. W'. Thornthwarte a partir des températures moyennes mensuelles, par le truchement des indices therniques mensuels et annuels, et comple tenu de la latitude et du nombre de jours de chaque mois. (Exapotranspirometre: ase lysimétrique a niveau constant; pluviomètre, thermomètre; formules diverses, formule de C. W. Thonsmwarte, choix de la pérlode convenable pour dresser un bilan valable de l'eau, ajustement par annia.)

Bilan de l'ean dans le sol (au point de vue agricole). - Le tableau III donne ce bilan pour la station d'Angoulême :

T A B L E A U I I I

Angovlême (Altitude : $82 \mathrm{~m}$ ), - Eléments mensuels nu bran de l'Eau du sol (mm)

\begin{tabular}{|c|c|c|c|c|c|c|c|c|c|c|c|c|c|}
\hline & $\stackrel{\dot{\Xi}}{m}$ & 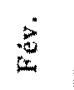 & $\stackrel{n}{\stackrel{n}{z}}$ & $F_{5}^{5}$ & $\ddot{E}$ & $\Xi$ & $\bar{\Xi}$ & 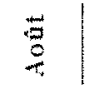 & $\underset{\vec{G}}{\dot{a}}$ & $\stackrel{\Xi}{\Xi}$ & $\ddot{b}$ & $\stackrel{\leftrightarrow}{\mathscr{U}}$ & ANNËE: \\
\hline $\begin{array}{l}\text { 1. Evapotranspiration poten- } \\
\text { tielle: }\end{array}$ & 11 & 16 & 31 & 51 & $8 \frac{1}{4}$ & 100 & 127 & 115 & $8 s$ & 50 & 24 & 14 & $n=715$ \\
\hline 2. Précipitations : & 58 & 66 & 69 & 64 & 70 & 74 & 51 & 55 & 56 & 80 & 79 & 82 & $p=804$ \\
\hline $\begin{array}{l}\text { 3. Vaxiation de la réserve d'eaul } \\
\text { du sol: }\end{array}$ & & & & & 14 & -35 & -51 & & & 30 & 55 & $15 i$ & \\
\hline 4. Réserve d'eau utile: & 100 & 100 & 100 & 100 & 86 & 51 & 0 & 0 & 0 & 30 & 85 & 100 & \\
\hline $\begin{array}{l}\text { 5. Evapotranspiration effective } \\
\text { (actual evapotranspiration, } \\
\text { de Thornthwate) : }\end{array}$ & 11 & 16 & 31 & 51 & 84 & 109 & 102 & 55 & $56 !$ & 50 & 24 & 14 & $r x=003$ \\
\hline $\begin{array}{l}\text { 6. Déficit (water deficiency, } \\
\text { de Thornthwaite) : }\end{array}$ & & & & & & & $2 \%$ & 60 & 27 & & & & $d=112$ \\
\hline $\begin{array}{l}\text { 7. Excédent (water surplus, } \\
\text { de Thornthwaite) : }\end{array}$ & 47 & 50 & 38 & 13 & & & & & & & & 53 & $s=201$ \\
\hline $\begin{array}{l}\text { 8. Ecoulement (runoff, } \\
\text { de Thornthwaite) : }\end{array}$ & 37 & 43 & 41 & 27 & 14 & 7 & 3 & 2 & 1 & 0 & 0 & 26 & 201 \\
\hline $\begin{array}{l}\text { 9. Différence entre les précipi- } \\
\text { tations et l'évapotranspira- } \\
\text { tion potentielle : }\end{array}$ & 47 & 50 & 38 & 13 & -14 & -35 & & & -27 & 30 & 55 & 68 & 80 \\
\hline $\begin{array}{l}\text { 10. Coefficient mensuel d'humi- } \\
\text { dité (moisture ratio, } \\
\text { de Thornthwaite): }\end{array}$ & 4,27 & 3,12 & 1,23 & 0,25 & $-0,17$ & $-0,32$ & $-0,60$ & $-0,52$ & $-0,38$ & 0,60 & 2,29 & 4,85 & \\
\hline
\end{tabular}


1" Si les precipitations du mois (2) sont supérieures a l'évapotranspiration potentielle (1):

a) L'évapotranspiration réelle (5) est égale a l'évapotranspiration potentielle;

b) L'exeédent (3) est emmagasiné dans le sol (4) jusqu'à saturation (ThonNrHwarte admel $100 \mathrm{~mm}$. compte tenu de la réserve antérieure du sol; (iEsLA domne $200 \mathrm{~mm}$ pour la France);

c) La partie de lexcedent dépassant éventuellement $100 \mathrm{~mm}$ constitue le water surplus (7).

2: Si les précipitations du mois (2) sont inférieures à l'évapotranspiration potentielle (1), l'évapotranspiration réelle (5) comprend:

- Les précipitations (2);

-. Tout ou partic de la réserve du sol it).

a) Si la réserve antérieure du sol (4) est suffisante pour combler l'insuffisance des précipitations (5), l'évapotranspiration réelle est encore égale à l'évapotranspiration potentielle; l'excédent éventuel les réserves (4) reste emmagasiné dans le sol, jusqu'a concurrence de $100 \mathrm{~mm}$ et sa partie dépassant éventuellement $100 \mathrm{~mm}$ constitue encore le water surplus;

b) Si la réserve du sol est insuffisante pour parfaire l'évapotranspiration potentielle, l'évapotranspiration réelle reste inférieure à celle-ci, la réserve du sol est épuisée et la différence constitue le «water deficiency» (déficit agricole). Cet état est atteint en juillet dans l'exemple d'Angoulême.

$3^{*}$ Pour amoreer le calcul, on choisit le mois ou la réserve de l'eau dans le sol est à son maximum, par exemple à la fin d'une période où les précipitations ont été supérieures aux besoins (avril dans l'exemple d'Angoulême).

4" La moitié de leau susceptible de s'écouler au cours d'un mois (water surplus 7 ) est entrainée dans le courant du mois et constitue I'écoulement réel (runoff 8), l'autre moitié étant retenue jusqu'au mois suivant. L'amorce du calcul se fait ici à partir du premier mois présentant un excédent wa ter surplus). C'est le mois de décembre dans l'exemple d'Angoulème. Le total annuel du water surplus (7) est égal au total annuel du runoff (8).

5. La différence entre les précipitations et l'évapotranspiration potentielle (ligne 9) permet de calculer le coefficient mensuel d'humidité (ligne 10), quotient de cette diffẻrence par l'évapotranspiration potenfielle.

Les quatre criteres de rlassificalion des climals, d'après ThonNthwalte, sont :

A. -.. L'indice global d'Hummté (moisture index) :

$$
\begin{aligned}
\mathrm{I}_{m} & =\mathrm{I}_{h}-0,6 \mathrm{I}_{a} \\
\text { avees: } & \mathrm{I}_{h}=100 \mathrm{~s} / n \text { (rapport d'humidite) } \\
\mathrm{I}_{n} & =100 \mathrm{~d} / n \text { (rapport d'aridite) }
\end{aligned}
$$

d'où :

$$
\mathrm{I}_{n}=\frac{100 s-60 d}{n}
$$

$s=$ water surplus annuel,

$d=$ water deficiency annuel,

$n=$ water need annuel (évapotranspiration potentielle).

Thonvrhware admet ainsi qu'un excédent de $6 \mathrm{~mm}$ d'ea u dans une certaine saison peut suffire à annihiler les effets eventuels d'un déficit de $10 \mathrm{~mm}$ dans la saison suivante: sous l'influence de l'humidité du sous-sol ou des nappes d'eau souterraines, la transpiration conti- nue, mais a un rythme plus lent. Thonntrwarte définit ainsi neuf types climatiques, allant du type aride :

$$
\mathrm{E}\left(-60<\mathrm{I}_{m}<-40\right) \text { au type perhumide }\left(\mathrm{I}_{m} \geqslant 100\right)
$$

Les cartes 4 à 9 établies pour la France par Marcel Gannien, d'après les moyennes de température et de précipitations, à trois cent trente-six stations, pour la période 1891-1930 indiquent, dans l'ordre, la répartition :

-De l'évapotranspiration potentielle moyenne annuelle (fig. 4 ci-dessous);

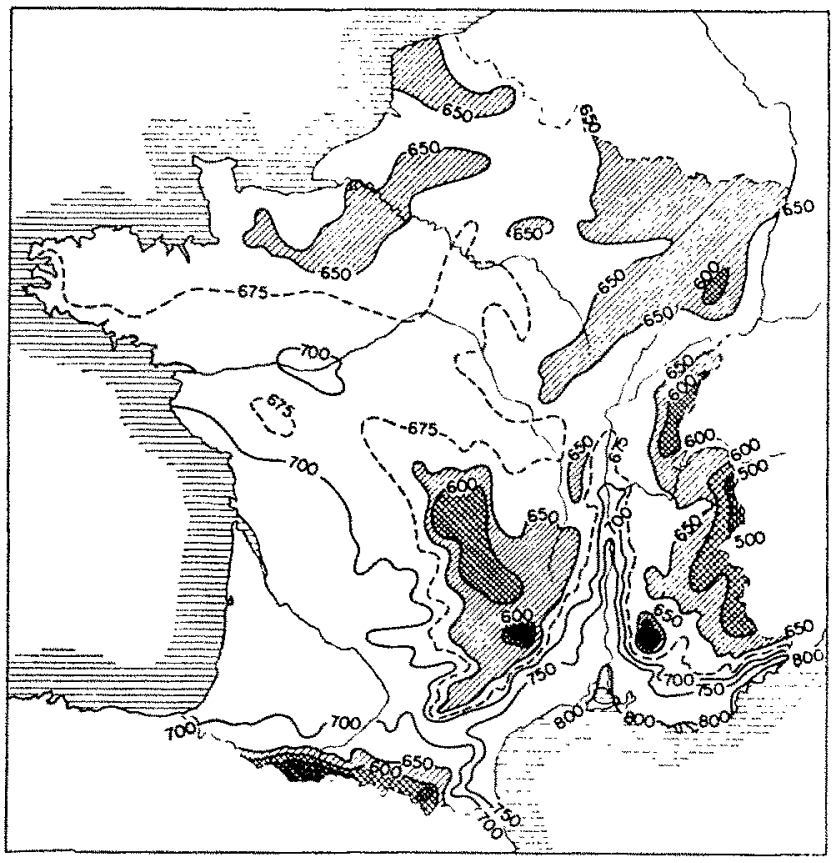

CARTE 4

Evapotranspiration potentielle en $\mathrm{mm}$.

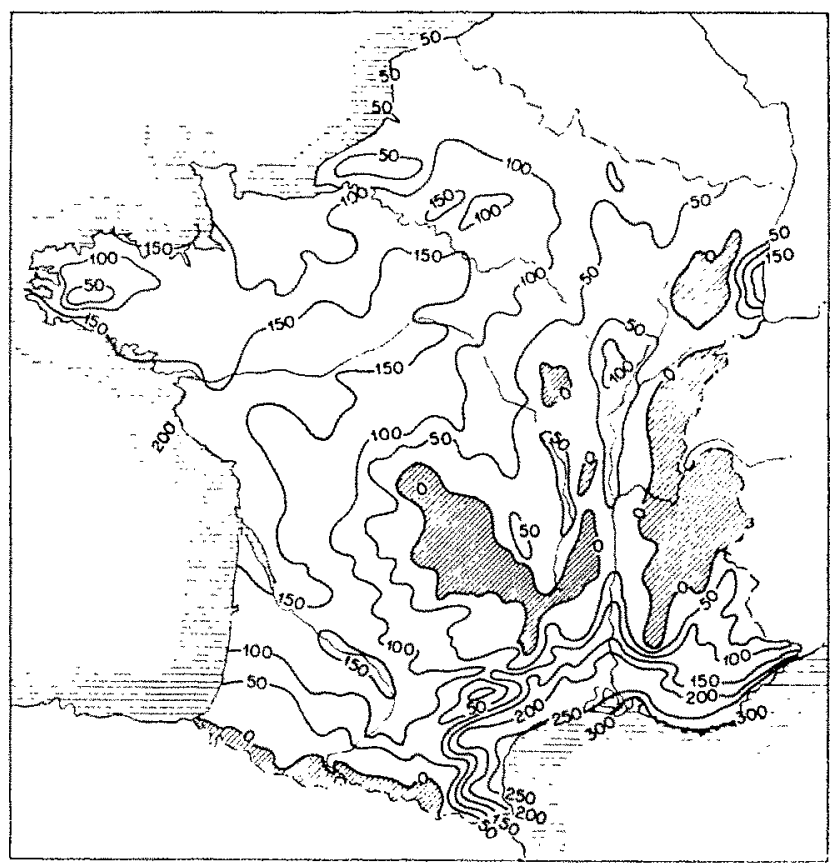

Cante ?

Water deficiency de C. W. Thornthwaite. Defficit moyen annuel d'ean. (au point de vue agricole) 
- De l'excédent total annuel d'eau (fig. 5);

- Du rapport d'humidité (fig. 6);

- Du déficit total annuel d'eau (fig. 7);

- Du rapport d'aridité (fig. 8);

- De l'indice glohal d'humidité (fig. 9).

Nous regrettons vivement de ne pouvoir donner ici. faute de place, ces intéressantes eartes. Nous nous bornerons a reproduire les cartes 4 et 7 qui intéressent particulièrement les hydrologues et le diagramme 10 qui illustre le tableau 3 ci-dessus.

Le déficit de 150 à $200 \mathrm{~mm}$ donné pour la vallée de la Loire par la carle 7 a surpris $M$. Thonsruwarre: il peut cependant s'expliquer d'une part, par les secheresses estivales assez fréquentes dans celte région et par Ia partie importante plantée en vignes, dont les exigences en cau sont relativement faibles, d'autre part par les réserves profondes du réseau hydrographique des vals de Loire, sans doute insuffisamment estimés à $100 \mathrm{~mm}$ dans la formule de Thonsthwarte;

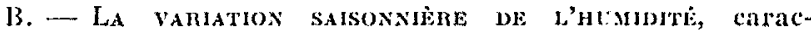
térisée :

a) Poux les climats humides $\left(\mathrm{I}_{m}>0\right)$, par la variation saisomnière du rapport d'aridite, on du water deficiency,

b) Pour les climals sees $(I,<0)$ par la variation saisonnière du rapport dhumidite ou de l'excedent (water surplus);

C. - Linnce v'erfotate thennove, d'apres l'évapotranspiration potentielle (fig. 4) : neuf types climatiques allant du gel $(n<142 \mathrm{~mm})$ au climat mégathermal $(n>1.140$ mm). La France se classe soit dans la catégrie $C^{\prime}$, du climat microthermal $(427<n<570)$ soit, surtout dans la catégorie $B_{1}^{\prime}$ du elimal mesothermal $(570<n<712)$;

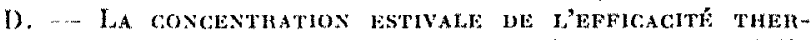
angue, rapport de l'évapotranspiration potentielle des trois mois les plus chauds au folal annuel des besoins en eau (huit classes).

Le elimat d'une station peut, dans ces conditions, etre défini par un symbole comportant quatre lettres pourrues ou non d'un indice (par exemple, pour Angoulême : $\left.C_{2} B_{2}^{\prime} r b_{4}^{\prime}\right)$.

\section{Conclusion}

Il est regreltable que lauteur n'ail pas pu tenir comple du déficit de saturation ou de la vitesse du vent, de laugmentalion de l'évaporation par decroissance de pression avee laltitude.

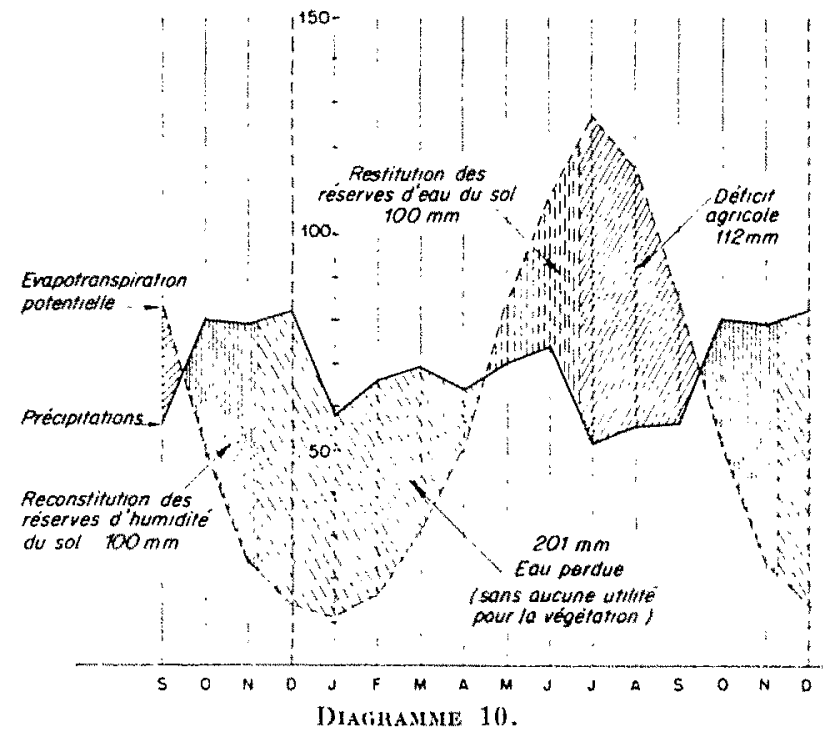

Bilan motyen annuel de l'ean (Angouleme: 1891-1930).
Cependant les agronomes apprécient la simplicite de la representation graphique du bilan de l'ean du sol (fig. 10), qui éroque celle que $A$. Cotratix: utilise couramment.

Les hydrotechniciens peuvent, de leur colé, compares les valeurs de leur propre «déficit d'écoulement» calculées par différence entre les précipitations el les débits, avec les différenees des nombres indiqués par les cartes 4 et 7 pour une mime region (water need water deficiency). On pourrait, de mème, caleuler ce défcit d'ecoulement par différence entre les nombres d'une carte pluviometrique (non comprise dans le memolre) et ceux de la carte 5 (water surplus s) pour une mime région.

En effet, on remarquera que l'on a, pour l'éraporation réelle, a l'échelle annuelle $(x$. tabl. Inl):

$$
\begin{aligned}
& a r=1 t-d \\
& 603=715 \cdot-112
\end{aligned}
$$

ou cheore:

$$
\begin{aligned}
& e \cdot r .=p-s \\
& 603=804-201
\end{aligned}
$$

\section{Discussion}

11. le President remereie M. Anssur d'avoir expose, dune facon claire, comment on dresse un hilan d'boulement, suivan la méthode Thonxtuwates qui, fout en étant d'inspiration agronomique, peat atssi hien s'appliquer aux études hydro-mécaniques.

M. Ropma souligne que pour l'etablissement thes cates de deficit d'eoulement presentees par M. AnLFny, on a dû, par suite du grand nombre de stations, considerer d'une part les $1 / 12$ corrigés de levapolmaspiralion annuelle (somme des indices mensuels), dautre parl les moyennes mensuelles interamuclles de l'eoulement: celte méthode de calcul lend à surtestimer les deffeits d'écoulement. On aurait peut-être trouré des résultals légèrement différents en opérant uniformément pour chaque anne dobservations sur les douze indices mensuels de l'évapotranspintion. Malloweusement, cette méthode, plus exacte, aurait conduit a multiplice les opérations dans des proportions lelles que la mise an point des cotes de deficit aurait exigé, sans donte, des delais prohibitits.

M. ArLeny croit toutefois que les rasomnements de Thonvтrwarte ne sont pas applicables sur une anne.

M. Tunc rappelle que le type dindice climatique ulilisé par Thonnthwarte est bien connu des agronomes: c'est ainsi qu'en 1937 Gest.n et Servy ont proposé daus un article des Annales Agronomiques un indice de secheresse calcule mois par mois et cortelatif de l'humidita du sol; d'autre part Penman en Augleterre, Henin et ses collaborateurs en France, calculont les bilans a peu pres

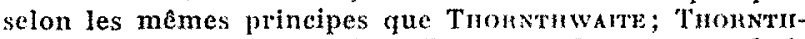
warre a le merile d'avoir diffuse ces inecs. On souhaiterait d'alleurs des methodes de calcnl plus simples. telles que celle du comte de Gispsms el cello que le Laboratoire des Sols à Versailles cludie sous la direction de M. Hexis. Cependant la valeur de $100 \mathrm{~mm}$ admise par ThonNTHWate comme emmagasíncment maximum de l'eau dans fe sol semble ponvoir être acceple comme moyenne générale de valeurs locales pouvant aller de $50 \mathrm{~mm}$, dans les terrains perméables a climat pré-desertique et a fable végétation, à 200 et $300 \mathrm{~mm}$ d'après M. Coutagne et M. ne Montmarex. Dans le cas de terrain a nappe phreatique proche de Ja surface ton peat rencontrer des valeurs encore plus eleveos.

M. Capres rappelle qu'il a tronve $200 \mathrm{~mm}$ en 1952 a Alrance. 
Répondant à une question de M. Bounner, M. AnLery dit que la méthode ThonNthwarte ne pourrait etre appliquée en France méridionale qu'après une correction deduite, non d'extrapolation ou d'interpolation entre les constantes proposées pour les Etats-Unis, l'Australie ef l'Angletere ou la France, mais de mesures ayant un caractère local plus serré. M. Thonnthwarte, d'ailleurs, serait tout disposé à collaborer à ces travaux de généralisation.

M. le Président indique que les applications de la méthode Thonverwatre à certains bassins de France et d'Afrique du Nord imposent des volumes mis en réserve dépassant les $100 \mathrm{~mm}$ prévus par Thonstrwarte.

4. le Président rappelle en concluant que M. Artiny a très bicn insisté sur lopposition entre le déficit d'écoulement intéressant les hydro-mécaniciens et le déficit d'évaporation interessant les botanistes et les agronomes : les cartes qu'il a dressées, notamment celles qui permettent de calculer le déficit d'écoulement, sont très intéressantes.

\section{COMMUNICATION DE M. RAVIER}

Ingénieur, Chef du Sernice «Hydrologie » à la R.E.H. Pyrénés-Atlantique d'Electricité de France

\section{LE GAVE DE SAINTE-ENGRACE ET SES APPORTS SOUTERRAINS EN AMONT DU BARRAGE DE L'USINE DE LICQ-ATHEREY}

L'exposé de M. Ravier devant avoir une suite à la prochaine session, il sera rendu compte de l'ensemble de son étude dans un numéro ultérieur.

Commentaire de $M$. le Président.

M. le Président rappelle qu'il a suggéré à M. Ravien cette intéressante communication, et le remercie vivement.

Vu l'heure tardive, les membres ayant des questions a poser ou des remarques a faire sont priés de les adresser par écrit au Secrétariat.

\section{SÉANCE DU JEUDI APRÈS-MIDI 18 JUIN 1953}

La sétnce est preside par M. Ganme, Président de la Section « Machines $\gg$.

COMMLILCATON DE HM. L. ESCANDE ET J. NOLGARO

\section{ÉTUDE THÉORIQUE ET EXPÉRIMENTALE DU FONCTIONNEMENT DES CANAUX DE FUITE EN CHARGE, EN L'ABSENCE DE CHEMINEE D'ÉQUILIBRE.}

2" Partic : PRISE EN COMPTE DES PERTES DE CHARGE, CAS D'UNE FERMETURE

NON INSTANTANEE, ETUDE SUR MODELE REDUTT ET YISUALISATION DE LA CAVITATION

Le mémoire de MM. Escanne et Noctano a été publie dans le numéro $5 / 1953$ de la llonille Blanche.

\section{Discussion}

M. Le Président remercic M. Escande de sa communication et souligne le résultat suivant: l'influenec de la perte de charge sur le fonctionnement du canal de fuite en charge, en labsence de cheminée d'équilibre est, dans les cas moyens, très fable. Il rappelle que le film projete illusire la formation de la poche de cavitation, sa fermeture et son amortissement.

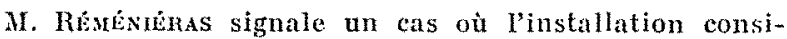
dérée par .I. Escande n'est pas protégée par la présence d'un clapet : ouverture du distributeur de la turbine pendant la remontée de l'onde, consécutive a une fermeture antéricure du dit distributeur; le choc des deux veines à vitesses opposées - l'une venant de l'usine, l'autre réfléchie à l'extrémité aval du canal de fuite - crée une surpression assez difficile à ealealer en grandeur et en durée, mais que l'on peut considérer comme une fonction de la somme des vitesses moyennes de ces deux veines : dans cette hypothèse, et dans le cas étudié par M. Escanise, cette surpression atteindrait 200 metres d'eau environ dans un canal calculé pour une pression d'une dizaine de mètres en moyenne. Quelle est la probabilité de réalisation de la succession de telles manourres en pratique?

D'autre part, M. Rónciśnas remarque que dans le film présenté par M. Escande, la cavilation est caracteriséc par la formation d'une série de bulles, et non d'une poehe d'air unique comme prévu par la theorie; cela pourait ètre dû à la résistance de leau a la traction; cette résistance est d'autant plus considérable que l'eau est pure; par contre, l'existence dans l'eau de bulles d'air cu d'autres noyaux diminue cette résistance et favorise la naissance d'une cavitation, localisec au début aux points fables ci-dessus sous la forme de bulles, puis générallisée si l'effort de traction sur l'cau devient très grand.

M. Bencision s'intéresse virement à cette question de cavitation beacoup plus iréquente avee les pompes qu'avec les turbines: il se demandait si, dans le eas d'une fermeture instantanéc, l'arrêt du refoulement se traduit par une carilation en masse, occupant foute la section, et qui, dans le cas d'une fermeture instantanee, serait visible au cinema sous la forme d'un \& piston * homogene se déplayant tans la conduite, ou par une bulle de vide occupanl seulement la partie supericure de la conduite horizontale ef cheminant depuis la vane jusqu'à l'extrémité. Le film de M. Fscaxine est done trés intéressant pour M. Burabaron car il lui montre que le phénomène se rapproche de la cavilation en masse arec un dégagement de bulles et une propagation limité probablement parce que lat fermeture de la furbine n'est pas instantanée.

De plus, éest Ja fermeture de la poche qui, plus que ha cavitation elle-même, donne des inquiétudes. Il s'agirail done de savoir où et comment se ferme la poche, afin de déterminer l'emplacement du elapet; or dans le eas des pompes le problème se complique du fait de la plus longue clurè du phéhomène, de la longueur el du profil complexe des canalisations de refoulement, toujouts plus ou moins inclinées.

Les ruptures de pente créent, en effet, des points sin suliers où peuvent se former des bouchons d'eau dus aux variations de vitesse, d'où l'intérêt de poursuivre les rechercher avec des conduites inclines et ainsi que lo précise M. le Président, des fermetures instantanés.

Sur la demande de M. le Président, M. Escanoe préciso qu'il a étudié, il y a quelques années, la transmission des coups de bélier dans le cas de l'ouverture et de la fermeture d'une turbine place entre une conduite force et un camal de fuite en charge, mais sams arriver jusqu'a lhypothese de la cavilation. Le probleme de la cavita" tion trates aujourd'hn se rapporte an cas simplifte d'une variation linẹaire du débit. 
M. le Président remarque que la durée de fermeture de 18 secondes envisage par M. Escande n'est tolérable en exploitation que moyennant un déchargeur, que, d'autre part, du fait des faibles pressions qui régnent dans le canal de fuite, il peut se produire, à la suite de l'ouverture, une onde de retour qui pose un problème de cavilation.

\section{COMMENICATION DE MM. L. ESCANDE ET HURON}

\section{ÉTUDE THÉORIQUE DE LA STABILITÉ D'UN SYSTĖME DE DEUX CHAMBRES D'ÉQUILIBRE PLACÉES L'UNE A L'EXTRÉMITÉ AVAL DU CANAL D'AMENÉE, L'AUTRE A L'EXTRÉMITÉ AMONT DU CANAL DE FUITE}

Le mémoire de Ma. Escande et Hunon a été publié dans le numero $5 / 1953$ de la Houlle Blanche.

\section{Discussion}

M. le Président remercie M. Escande.

M. Mrren signale que le résultat obtenu par M. Escasbe concorde parfaitement avee ceux qu'il développera luimême dans une étude générale qui paraitra sous peu rans la Honille Blanche : il montrera qu'une installation hydroélectrique munie de deux chemines d'équilibre, l'une à l'amont, l'autre à l'aval des groupes, et munie d'un régulateur parfait (au sens de Thomal est ì peu près équivalente, du point de vue de ses ascillations infniment petites, à un ensemble de deux installations oblenues par la séparation de l'installation initiale en une partie amont et une partie aral. Ceci est vai si les pertes de charge sont petites devant h chute nette, ee qui est toujours le cas en pratique.

L'équation caractéristique des oscillations d'une ins-
Inllation a deux chemines est du quatrieme degre; si on lolere une erreur qui est au maximum de $t-h / h$ sur les coefficients de l'équation, celte equation so scinde en deux equations du deuxieme ordre dont chacune est relative a l'installation particlle oblenue comme décrit plus haut.

En particulier, les conditions de stabilite sont les denx conditions de Thoma relatives à chaque section, et les pseudo-périodes d'oscillations sont celles qu'on calculerat en divisant l'installation en deux.

L'erreur maximum due à l'approximation décite plus haut est en général obtenue quand les deux sections sont juste égales $\dot{x}$ la section de thoma correspondante, comme ceci apparaît dans le cas des exemples numériques dooisis par M. Escanor. Lapproximation dont M. Mryen a parlé est daulant plus justifie que les coefficients de l'equation caractéristique ef surlout eertaine: quantites dont ils sont fonction (par exemple la valeur de la perte de charge) ne sont en genéral pas conmus avee une precision aussi grande que lapproximation consentic.

M. Bouvand signale qu"on a l'impression, quand les deux périodes d'oscillation sont sensiblement identiques, que le phénomène sera sensiblement aggrave par rapporl a ce que donneraient les oseillations séparés des deux systemes et il semble que l'amplitude devait devenir considérable. Il semble, en lout cas, que cest dans ces conditions qu'elle serail maximum.

\section{COMMUNICATION DE MH. DANEL, DURAYI ET CONDOHIOS}

\section{INTRODUCTION A L'ÉTUDE DE LA SALTATION}

Le memoire de MM. Danel, Drmaxd of Conmonos at sat discussion sont presentes dans le $n^{\circ} 6,1953$ de la Honille Blanche.

\section{SÉANCE DU VENDREDI MATIN 19 JUIN 1953}

La séance est présidée par M. Hepxen, Président de la Section Genic Civil at Conduites $\$$.

\author{
COMMLNICATION DE M. BIROT \\ professeur à la Fuculté des Letres de Lille
}

\section{PROFIL D'ÉQUILIBRE TRANSVERSAL DES LITS FLUVIAUX ET ÉROSION LATÉRALE}

\section{Résumé}

La section monille des cours f'eau ayant atleint leur profil d'équilibre longitudinal est toujours beacoup plus large que profonde; ce rapport varie entre 5 et 100. 1. Charge solide nulle:

$\checkmark$ max, correspond a $R_{11}$ maximum (roctangle de larse ur 13 double de la profondenr $H$, ou mieux, demi-eerele).

Celle seclion dable tend a s'bargir jusqu'ì ce que le gradient de $V$ entre le centre ef les parois diminue tellement que la capacité érosive des coumants de turbulence solt très attenué. D'où tendanee a $\mathrm{B} / \mathrm{H}>2$. $2^{\circ}$ Aver charge solide:
Gubent determine le profil de section mouillex oplinum pour le transport (capacite maximum) mats ses experiences sur canaux ne liennent pas comple de l'érosion des parois; lo mpport $H / B$ est d'autant plus grand que la pente et le débit sont plus élevés ef les alluvions plus fines. 11 varie tulye $1 / 2$ et $1 / 30$. Deux effets antagonistes : un dargissement du chenal augmente la capacite (par aceroissement de la surface de traction el diminution des froltements sur les parois laterales mais dimmue considerablement la vitesse (en rason de ha reduction de la profondeur).

La puissance de transpont varie comme $v^{3}$, eube de

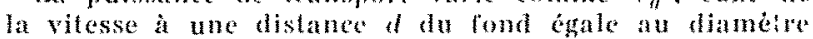
moyen des particules entaineses: or, $V_{d} / V_{m}$ varie comme log $(d / R)$ (rox kathax) ou comme $(d / R)^{1 / 4}$

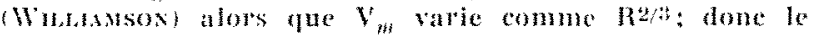
transport (roulement ef salfation) par wille de surface

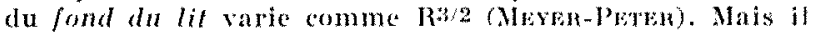
s'agil seulement de la surface da fond du lit, les courants de turbulenee provenanl des parois elant steriles au point de vue du transpost.

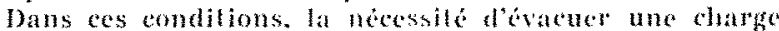
solide donnte pent imposer an lil whe largeur tres supé rieure a celle qui correspond au ragon lyedratique maximum.

Lapplication des formules de Mryen-Peren a un confluent montre que, des quon a affatre a des rivieres 
fortement chargées, il est nécessaire que $\mathrm{B} / \mathrm{H}$ augmente après le confluent.

Nombre de solutions possibles d'aprés la formule MeyenPeren :

Dans le cas simplifiè où la rugosité des parois égale celle du fond :

$1^{\circ}$ Il existe pour $d_{m}$ Q et 1 donnés une seule section monillée telle que le débit solide soit maximum.

2" Il existe pour $1, Q$ et $G$ donnes, au moins deux sections mouillées plus larges que profondes permettant d'évacuer le débit solide.

En général :

En explicitant dans la formule de Mexen-l'exwn le debit d'après la formule de Stricklen-Manning (en fonction de R et $a=H / B$ ) on établit l'équation de la dérivée du débit solide et l'on trouve, par résolution graphique, que le débit solide est maximum pour $\left(k_{s} / k_{r}\right)^{3 / 2}$. IR $>A d(1)$ correspondant à une valeur de IR peu supérieure aux conditions eritiques d'entrainement. Cela prouve qu'il existera toujours un rapport optimum $H / B$ et pour une valeur donnée du débit, il y aura deux formes du lit possibles dans la section ou $H / B$ est constamment inférieure $1 / 2$. Il faudrait $y$ ajouter les autres solutions possibles pour $H / B>1 / 2$, cas très rare dans la nature.

La discussion de cette résolution graphique montre que plus la pente et le débit. sont forts pour un calibre donné, plus $\alpha$ est petit. Par contre les cours d'eau dont la capacité de transport unitaire dépasse de peu la puissance critique d'entrainement adoptent la section la moins large possible (rayon hydraulique maximum pour assurer au moins le débit d'entraînement). Dans cet intervalle $B$ décroit de plus en plus lentement avec $R$.

Ces considérations théoriques expliquent que le rapport $B / H$ est d'autant plus grand que le matériel transporté est plus abondant et plus grossier, ce qui a été exprimé, pour les cananx d'irrigation de l'Inde, par ane formule empirique :

$$
\mathrm{B} / \mathrm{H}=\mathrm{A} \mathrm{Q}^{1 / 0},\left(w, \mathrm{G}_{s}\right)^{7 / \mathrm{H}}
$$

$\mathrm{G}_{s}$ étant la charge par unité de volume liquide et $w$ la vitesse de chute des particules. A partir du moment où une forte proportion des débris est prise en suspension, les courants de turbulence qui naissent sur les parois ne sont plus stériles, mais participent à la sustentation des debris.

Vartations de $\mathrm{B} / \mathrm{H}$ le long des cours deat naturels:

Section amont: Cycle d'crosion jeune. $\mathrm{R}$ se rapproche de la valeur maxima; les alluvions sont grossières mais assez peu abondantes. $H / B$ peut être plus grand que l'unité au voisinage de chutes importantes (circulation torrentielle, tourbillon d'axe horizontal, perpendiculaire a la direction du courant et avec cavitation).

Section moyenne: $\mathrm{B} / \mathrm{H}$ augnente rapidement (prise en charge de masse rroissante de débris moins grossiers sc déplaçant surtout par roulement et saltation).

Vers l'aval: B/H diminue de nouveau (constaté sur les rivières aux U.S.A.) parce que la plus grande partie des débris est prise en charge par suspension : les courants de turbulence nés sur les parois participent à cette action. En fait, il n'en est pas toujours ainsi (phénomène aberrant do au remblaiement d'origine eustatique qui affecte toutes les rivières du globe jusqu'a une distance plus ou moins grande de l'embouchure).

Une conséquence de ce principe : le $B / H$ des rivières calcaires est faible, en rapport avec la charge transportée.

(1) Bien que cee ne soit pas explieité dans le memoire, nous domnons les deffntions suivantes des symboles, diapres METEF-PETER :

$I=$ pente moyenne de la ligne d'energie:

$k_{r}=$ coemcient de rugosité des grains;

$k_{s}=$ coemcient de rugosité du lit.

\section{Phénomènes morphologiques et climatiques favorisant l'érosion la térale}

$1^{-}$Rivières coulant au pied des montagnes: ces dernières fournissent des alluvions grossières, lourdes et se décomposant difficilement à la zone de piedmont, formée elle-même de roches tendres : vitesse des couches supérieures élevé, attaquant les roches tendres des parois, alors que le fond du lit est pavé.

$2^{\circ}$ Variation $d u$ débit lituide: lors des crues, une rivière de régime irrégulier crense les monilles et sape les parois; lors des basses eaux, les creux sont comblés, mais le recul des parois est un fait irréversible. Ainsi la rivière tendra toujours à adopter la plus large section mouillec possible.

$3^{\circ}$ La migration laterale du lit mineur est favorisée par une combinaison des deux proeessus précétents. Pendant les maigres, le eentre du lit est légèrement bombé et la rivière se réfugie dans une rainure contro les parois de roche tendre qu'elle fait reculer, ef où elle s'enfonce plus facilement que les alluvions. Mais a la fin de la crue, ce nouveau sillon est pavé a son tour.

Enfin, le pavage du fond du lit est porté d'autant plus loin vers l'aval que le débit est plus variable.

$4^{\circ}$ L'érosion latérale des rivières à méandres est réalisée en situation d'équilibre dans des caux peu turbulentes : l'alluvionnement de la rive convexe represente un volume approximativement égal au creusement de la rive coneave.

\section{Discussion}

M. Dupuy ue croit pas que, dans le cas d'entrunement par charriage des alluvions sans cohésion (sables of calloux), la condition de stabilite d'un lit de rivicre on de canal puisse s'exprimer en fonction du seul parimètre $H / B$, rapport de la profondeur a la largeur du lit. En effet, les formules empiriques ainsi établies conduisent à des écarts atteignant $800 \%$ pour le dimensionnement d'un même lit. D'autre part, $H / B$ n'est pas significatif de langle ou de la forme du falus, parametres qui sont fondamentaux dans l'étude de l'érosion latérale, c'est-à-dire de la stabilité des berges. M. Dupur a remarqué, dans une étude sur modèle, que des turbulences particulières prennent naissance dans les zones d'angles de l'écoulement, sans gu'on en puisse trouver trace ailleurs.

Par alleurs, la formule de MEYer-PBrar, dablie pour des canaux avec écoulenenl ì deux dimensions, est difficilement applicable, sauf cas particulier, à des vivières de profil quelconque, avec écoulement à trois dimensions. De plus, la dérivation de celte formule établie expérimentalement n'est pas sans présenter quelque danger.

Un procédé d'investigation qu'avait suggéré Lave, dail Vétude de la répartition de la force tractrice le long du périmètre d'un canal. M. Dupy: a procédé à diverses expériences: elles aboutissent à siluer les maxima de force tractrice dans le tiers inferrieur de la berge, alors que les érosions constatées ont liru dans le tiers supérieur. Ce seul aspect du probleme n'est donc pas suffisant non plus et la question apparait très complexe.

M. Bmor répond :

$1^{\circ}$ En tant que morphologue, il ne considère que l'état permanent d'équilibre, une fois obtenu l'érasement des parois. Les résultats proposés peuvent, en effot, ctre améliorés en considérant la géomélriv complèto du profil ot la turbulence sur les parois.

$2^{\circ}$ La formule de Meyen-Peren a élé appliquée au Rhin en amont du lac de Constance. Ses coeffelents ne paraissent pas chnnger suivant la nature des roches.

Sur la demande de M. le Président Barririon, M. Binot ajoute à son exposé la conclusion ci-après : le fait que pendant très longtemps le rapport $H / B$ d'une rivière 
diminue, puis de nouveau augmente légèrement vers l'aval peut se justifter par une considération théorique empruntee aux formules connues et implique aussi certaines exceptions comme la variante des rivières calcalies.

M. Ie Président Huren rappelle que les ingénieurs intéressés par la mesure du débit solide n'ont pas encore trouvé de procédé direct dans ee domaine et déterminent indirectement ce débit par des formules du genre de celles de Meyer-Peter.

Les incertitudes qui resultent de celfe carence apparaissent dans les traités de gégraphie qui reproduisent les résultats de ces mesures faites sourent par des procédés un peu simplistes. Le mérite de H. Bnot, e'est de fenter de jefer un ponl entre deux disciphines, celle des geographes et celles des hydrauliciens. M. le President émet le souhait que le contact qui a été pris aujourdhui soit maintenu el remercie M. Binor en lui demandant de continuer à apporter le fruit dic son experience el de ses réflexions à cette question.

Sur Ia demande de M. le President, M. Bunor donue te titre de son ouvrage: Problemes de morphologie ginerale.

\section{COMMIYTCTIOMS DE MM. MATTRE ET OBOLENSKI 1" NOTE SUR L'ÉCOULEMENT DANS LA PARTIE. AVAL DES EVACUATEURS DE SURFACE}

\section{par M. Matrue, \\ Ingeiniear an Laboratoire Vational dilgalranlique \\ 2" MESURES DE QUELQUES CARACTÉRISTIQUES DE L'ÉCOULEMENT DANS LA PARTIE AYAL DES ÉVACUATEURS DES USINES \\ DE SAINT-ÉTIENNE-CANTALES ET DE CHASTANG}

par M. Onolenski, Ingenient au Service des Eludes of Recherches Hydrauliques d'Electricilt de France

Ces deux mémoires seront publiess dans un prochain numbro de la Honille Blanche.

\section{Discussion}

II. Io President Hupwen felicite Ma. Marme et Onobexskt de leur dite interessante. I1 pense, an point de we terminologie, que le mol "ranne segment s convient micux que «anne secteur $»$ pour désiguer les vaunes co service it Saint-Etieme on it Chastang, lesquelles inferviennent par leur segment of mom par le plan du diedre. Puis, il demande s'il al éte fait d'autres essais comme ceux-ci en grandeur naturelle.

M. Matrue signale les essais de l'evacuateur de l'Aiglo realises il $y$ a quelques annes sur l'initiative de II. Corve, qui en a relate les résullats dans sa commu-

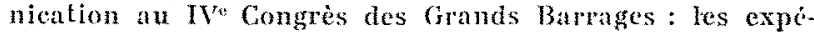
rimentateurs ne disposant pas d'appareillage special, le relevé des tirants d'eau avait été effectué le long d'un guideau, au moyen d'un fil it plomb. Ce moyen de mesure ne permet pas, compte tenu de la présence de la couche latérale émulsiomée, d'obtenir ume précision supéricure i $10 \%$, et le gonflement dù à l'émulsion donne lieu de penser que celte erreur est très probablement une erreur par exces, et, d'une erreur relative de $10 \%$ par excès sur le lirant d'eau résulle une erreur de $20 \%$ par défaul sup la charge an point considéré. Or, le resultat de ces mesures faisait apparafte a l'aval da coursier une charge réduite a la moitié de sa valeur initiale; la va leur corrigete de cette perte de charge serait done de 40\% de la charge totale au-dessus du point bas du coursier, ce qui diffère très peu de la valeur de $35 \%$ trouvé à Saint-Etienne-Cantalès pour un tirant d'eau relatil d/L légèrement plus fort.
M. Bounculaxox indique l'essai d'un Ingénieur améxicain, utilisant une poutre avec des touches pour déterminer le plan d'eau; cet essai peu réussi a’a pas ete publié.

Sur la demande de M. le Président, M. Marras précise que la vanne etait ouverte partiellement au Chastang, mais totalement à Saint-rienne où, toutefois, le plan d'eau avait été baisse pour éviter un trop grand dibit fin consequenes, la perte de charge mesure au Chastang comprenat forcément, outre le frottement sur le coursier, la perte singuliere a la traversée de la vame que lon peut, toutefois, considérer comme négligenble : l'ouvrage est bien tracé et l'econlement alimente par un plan dean ealme. En tout etal de fause, si l'on doil retrancher de la perle de charge nesure a Chaslang. la perte de charge singuliere due it la vame, les conelusions de ces essais, montrant les faiblesses des pedes de charge sur te coursier aux forts débits, sen trouvent renforcées.

Sur une question de M. Ie Préxident, relalive at la cerlitude d'avoir atteint les vitesses limites malgré le débil partiel, imposé par les circonstances, M. Majure répond que la vitesse est theoriquement constante a la surface de l'écoulement, quel que soit le débit, puisqu'clle dépend uniquement de la hauteur de chute sur lonvage, ì condilion, tontefois, que le débit soit supéricur an hebi eritique, e'est-à-dire que l'econlement en surface soit it potentiel; cette condition était remplie a Chastang, les essais ayant été rénlisés pour un débit double du débil eritique. Les vitesses mesures a Chastang par M. Omisssk s'etablissaient d'alleurs entre 27 et $27,90 \mathrm{~m} / \mathrm{s}$ suivant les sections, et encadratent la vitesse prevue par la loi de Bermouilli it 5 ot 10 of pris.

M. Habcibles s'inleressant à leffort sur lo fond M. Matre indique que leffort dirige vers l'aval, dú a lat traction de l'écoulement sur l'ouratge est exprime globalement, suivant te thereme des quantites de mouvement, par $\mathrm{Q} Q\left(V_{0} \ldots V^{\prime}\right)$ : $V^{\prime}$ etant la vitesse rélle a laval de l'ouvrage et $V_{0}$ la vilesse theorique en l'absence de froltement. Toutefois, la repartition de cet effort $F_{11}$ ke long du conrsier est difficile a étudier, la precision des mesures n'etant pas suffisante pour calculer exactement la rariation de guantile de monvement -.. ou la perte de charge - entre denx sections consécutives.

M. Marne pense qu'on a, d'aillenrs, tendance à exagerer l'importance de cel effort, en ce qui concerue l'érui-

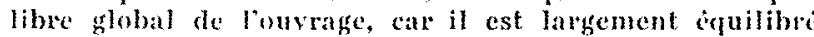
par la resultante des efforts de pression de l'éontement sur le coursier, résultante dont la composante lorizontale F.. est dirige vers l'amont; on a evalué ces deux efforts pour l'évacuateur de Bort a partir des mesures de pression et de ligne d'eau réalisées sur lo modèle : lordre de grandeur de $F_{2}$ est de 3.000 tomnes, contre 1.000 tounes seulement pour $F$. Par contre lorsque l'on considère l'équilibre de la partie aval du coursier, non solidaire du barrage ef reposant sur le toit de l'usine. il faut tenir compte de leffort de traction vers l'aval du au frotfement, car la résultante des forces de pression est pratiquement verticale dans celte partie de l'ouvrage rt n'intervient donc plus. Pour l'usine de Bort, on : mesuré directement sur modèle réduit, l'effort ulobal do traction vers l'aval résultant du froltement. Ces résullats ont conformé. dans ce cas, lévaluation approche obtenue a partir de l'evaluation de l'effort a haroi $\tau_{0}$ résultant de la formule de Manning-Stmocren. II est noter que cette concordance est probablentent due a ce que lécoulement est très peu différent d'un ecoulement pleinement turbulent a l'aval de lévacuateur de Bort, du fait de sa très grande longueur relative. II n'en serait pas nécessairement de mème pour un ouvrage plus court, sur lequel on peut penser que les efforts seraient plus faibles.

M. Lakezors remarque que l'energic dissipée à l'aval 
surpasse, d'après la communication de M. Marrae, les évaluations faites dans le passé. Une crue récente de la Dordogne ayant causé des dégâts à l'aval de Chastang, pose aux Services de l'Exploitation d'E.D.F. le problème de l'évaluation exacte de l'énergie restant à dissiper à la base du jet.

M. Marrne pense que c'est bien la que réside le prohlème essentiel, mais que les mesures, an ce domaine, sont pratiquement impossibles, à l'heure actuelle. En ce qui concerne Chastang, M. Matre pense que les dégâts causés par la récente crue de $1.700 \mathrm{~m}^{3}$ ont surtout mis en évidence l'importance de phénomènes secondaires:

- Ces dégats sont en effet localisés hors de la zone d'impact proprement dite qui, bien protégée, a convenablement résisté;

- D'autre part, ils sont dus surtout a l'importance de la crue dans un chenal non calibré pour un tel débit et a l'attaque des berges, peut-etre insuffisamment prolégeses, par lagitation de surface qui se propage vers l'aval a partir de la zone d'impact, a la manière d'une houle irrégulière et violente.

Sans insister spécialement sur le cas particulior de Chastang, cité à titre d'exemple, II. Lavalors pense, en tout cas, que tenant compte des remarques de M. Marthe, II $y$ aurait sans doute liea, dans l'avenir, d'apporter une plus grande prudence dans l'évaluation de l'énergic à dissiper à l'aval de ce genre d'ouvrage.

\section{SÉANCE DU VENDREDI APRĖS-MIDI 19 JUIN 1953}

La séance est présidée par M. Hujsar. Président de la Seclion «Genie Civil et Conduite».

\section{COMMUNICATION DE M. GRIDEL}

\section{A PROPOS DES I JOURNEES ITALIENNES DE L'HYDRAULIQUE " (Naples, mai 1953)}

\section{Résumé}

Les «Journees Italiennes de l'Hydraulique» qui ont lieu tous les ans dans une grande ville comportant une Université se sont déroulées cette annè à Naples les 1.1 el 15 mai, avec près de 250 participants. Linne prochaine elles auront lieu à Rome.

Les « Journees llaliennes de l'Hydraulique \$ sont organisées à l'echelle nalionale, mais comportent, toutefois, quelques participants étrangers, parmi lesquels avaient été invités cette année:

- La Société Hydrotechnique de France,

- Le Laboratoire National d'Hydraulique de Chatou (dn fait de la présence antérieure à ce Laboratoire de quelques stagiaires italiens, anciens elèves de Naples),

- M. Rovse, l'lydraulicien américain bien connu.

La S.H.F. avait chargé MM. Grubed et Manouenet, délégués par k* Laboratoire National d'Hydraulique, de la représenter.

M. Rouse n'a pas assisté à cette manifestation.

Cette année les «Journées Ttaliennes de l'Hydraulique» étairnt placées sous la présidence de M. oE Marchi, Professeur à l'Institut d'Hydraulique de l'Ecole Polytechnique de Milan, issisté de M. Iprorito, Directeur de l'Institut d'Hydratique de Naples.

Tout dabord, un hommage a été rendu a la mémoire de M. Scrumm, Directeur do l'Université de Padoue, recemment décéde, puis une Commission a été constitué pour s'occuper de la préparation du centenaire d'Allievi, qui doit avoir lien dans deux ans.

Dans l'ensemble, les communications présentées furent courtes; elles portèrent aussi bien sur des sujets intéressant la réalisation des ouvages, donc souvent extrahydrauliques (génie civil, mécanique des sols, etc.), que sur les questions liydrauliques proprement dites. Elles seront publices dans l'Fnergia Flellrick.

L'auditoire était composé de personnalités universitaires et administratives, ainsi que d'industriels. Les Societés de production electrique - même thermique daient amplement reprèsentes.
Les débats se sont quelquefois étendus a des questions de règlement administratif idiscussion sur les digues en (erre).

Les «Journées Italienmes de l'Hydraulique» ont été suivies de visites de laboratoires el de chantiers, ainsi que de réceptions par le Recteur ate l'Université, le Directeur de la Faculté, des Ingénicurs, la Société Méridionale d'Electricité.

Des déplacements louristiques ont été organises pour les participants le samedi $\mathbf{1 6}$ mai (Poestum, Amalfi, Sorrente) et tous les jours pour les dames qui araicnt accompagné les congressistes.

\section{SHANCES DE TRAVATR}

Le programme fixé pour les travaux, assez incompletem ment suivi, comprenait les sujets ci-apres : Entranement dair el de matériaux par les courants - Ecoulements à grande vilesse.

Trente-cing rapports onl até présentés:

a) Entrainement d'air et de materiaux par les conronts : à ce sujet ont participé :

- Le Laboratoire National d'Hydraulique, par une intervention de $M$. Manovenet sur les prises d'eau additionnelles;

- Mn. Gallo-Rotendi, par un travail d'ensemble sur le problène des débits solides;

- Mr. Contr, par une intervention tendant a traiter ce problème par sa mise en équation complete (M. wK Manchi a déclaré ne pas être d'accord sur ce point de vue);

- M. Manzolo: Aspect pratique des écoulements fluviaux et torrentiels;

- M. Graconelal : Electrophorèse pour consolidation des éléments fins;

- M. Rowita: Erosions au pied des piles de pont mal implantées;

- M. Barucherto : Vieillissement des canaux industriels;

- M. Indn : Capacité de transport solide des siphons.

b) Courants rapades : communications de :

- M. Mongiandini : Ecoulements de mixtures dans les conduites comportant des diaphragmes ef au passage des vannes;

- M. Vipanelli : Ecoulements torrentiels sur canal à $45^{\circ}$;

- M. Mamchetrr: Pertes de charge dans les tuyaux de zine.

Parmi les autres sujets traités on peut mentionner les communications relatives aux coups de bélier:

- M. Vrrx : Célériles ef surpressions dans les condnites à diamètre et épaisseur variables: 
- M. BÉsint : Considérations sur les cheminées d'equilibre avec etranglement à la base;

- M. Penart : Etude de l'onde positive ascendante proroquée par larrêt des turbines dans les camaux d'amenée avec interposition des clapets.

linfin, des communications suivantes sur des sujet, divers:

- N. Anuehas : Influence du champ de la pesanteur sur les écoulements dirigés vers le haut (essais effectués avec M. Scmem, à Padoue);

- Mr. Savastano: Mesure des vitesses el débits au moyen d'un champ magnétique;

- M. Fonnica : Pertes de charge aux variations de section en fonction des formes de raceordement;

-. M. Russo Spens: Percolation plane à surface libre avec capillarité

- 11. De Vro : Contróle par laboratoire de campagne d'un travail d'étanchéisation de piste d'aéroport;

- M. Mario VTT: Calculs relatifs au mouvement varic dans les écoulements en charge en tenant comple des frotiements.

\section{Visites}

Les participants onl visite le Laboratoire de l'lustitu d'Hydraulique de Naples où se trouvent des installations d'essais relatives aux mixtures, aux rannes automaliques at aux écoulements en genémi.

Nous avons également visité :

1" Le port de Naples.

a) En ce qui concerne la station de mesure de la hoult. Culle-ci comporte:

1. Des échelles à contacts ellectriques pour la mesure de l'amplitude sur la digue «duc des Abbruzes * de 20 en $20 \mathrm{~cm}$ et de 50 en $50 \mathrm{~cm}$ ),

2. Des dispositifs de mesure des pressions tous les $2 \mathrm{~m} \mathrm{de}+4$ an pied de la digue situc a $-12 \mathrm{~m}$ ).

3. Cne station de prise de rues stéréo-photogrammétriques disposece au fort Saint-Elme, qui domine le port;

b) Le chantiar de construetion de la nouvelle et très importante forme de radoub.

2" Le Consortium de Distribation des Eatar de Naples dépendant directement de la Caisse du Midj, qui s'equipe actuellement pour poupoir distribuer $600 \mathrm{~J} /$ jour d'eau par labitant.

En raison de la topographie de la ville, les installations se frourent abblies a cind niveaux differents et les noureaux réservirs sont aménagés à meme le tuf.

3" En Sicile (Ente Siciliano di Elettricita)

a) Le mannage d'Axcipa limitant un réservoir dont la capacile est de 30,4 millions de m" dont 27.8 utiles collectes sur un bassin rersant directement tributaire de $51 \mathrm{~km}{ }^{2}$ complete par des adductions secondares intéressant $52 \mathrm{~km}=$. La puissance installée est de $33.000 \mathrm{~kW}$ (dèbit de $9 \mathrm{~m} / \mathrm{s}$ sous une hauteur de chute de $417 \mathrm{~m}$ ) (turbines Pelton). L'ouvrage a nue hanteur de $103 \mathrm{~m}$, un développement de $252 \mathrm{~m}$ el une base de $94 \mathrm{~m}$; les pentes amont et aval des parements sont de 0,45 ; exéculé suivant les plans de M. Cl. Mancesreo, il comporte neuf plots évidés; l'économie de poids realise sur la totalite de louvrage est de $30 \%$. L'elancheite des joints reliant les plots entre eux cst assuré par un couvre-joint de ciment armé recouvrant des feuilles de rerre-asphalte, nue cavite remplic de bitume réchaufé électriquement et une lame de cuivre.

L'évacuateur de crues est prévu pour $1.500 \mathrm{~m} 3 / \mathrm{s}$, soit 20 a $25 \mathrm{~m}^{3 /} / \mathrm{s}$ par $\mathrm{km} 2$ de bassin versant. 11 comprend :

- En évacuateur en surface, compose:

$1^{\circ}$ De trois seuils deversants;

$2^{\circ}$ D'un dérersoir central obturé par une vanne levante et surmonté de clapets supérieurs; $3^{\circ}$ De deux déversoirs latéraux, in la naissance desquels sont deux vannes levantes.

- The vidange à mi-hauteur.

Ces deux ourrages evacuent dans une galerie de dicharge de $250 \mathrm{~m}$ a écoulement libre avec renillard.

La galerie damence a la centrale de Troma a une Iongueur de $7 \mathrm{~km}$, la conduite forces, en beton précontraint, un dianelre de $2,66 \mathrm{~m}$.

b) Banhate 1): Geta, construit en enrochements rangés avec masque d'étanchéité en ciment armé a tamont, parafouille, joints, ete. (hauteur $38 \mathrm{~m}$; longueur du conronnement $300 \mathrm{~m}$; ejaisseur a la base $80 \mathrm{~km}$; épisseur at sommel $6 \mathrm{~m}$ ).

L'un el l'autre de ees ourrages semblent devoir presenter a bref delai des problemes dengravement des retenues.

\section{Discussion}

En remerciant M. Gmbed, M. Le President remarque la forme el l'importance de l'evacuateur de crues du barmage d'Aneipa, qui est préru potr un débit de $1.000 \mathrm{~m} 3 / \mathrm{s}$, soit $20 \mathrm{~m}^{3} / \mathrm{s}$ par $\mathrm{km}^{2}$ de bassin versant.

M. Gnons. indique que ce dehit parait naturel an matite de l'ouvre, ru la violence des orages en Sicile.

N. Bouvan remarque que le principe des batrages evides est connu depuis assez longlemps et qu'il a di utilisé frequemment en Suisse (Barrage de la GrandlDixence), en Angleterre, ef qu'en France le harage projoté de Plan d'Amont est précisément de ee type (les alveoles sont ouvertes vers laval).

M. Ie President croit savoir que les Suisses ont envi satgé après coup de remplir particllement en béton leurs barages evidés par crainte d'un danger excessif en cas de bombardement.

M. Gnmer ajoute qu'en lialie l'Administration Mililaire controle tous les projets de barrage: a Ancipa, elle a fait ajouter la vidange intermediare qui n'etait pas prévue dans le projet initial. D'ailleurs, le risque d'une catastrophe humaine en eas de destruction de l'ouvage est très réduit a Aneipa, par suile de la nudite absolue du lerain 5 su $200 \mathrm{~km}$ i l'aval.

COMMUMLATHOY DE M. GHBRAT

\section{PREMIERS CRITÈRES DE COMPARAISON DES DIVERSES TURBO-MACHINES AU POINT DE VUE DES USINES MARÉMOTRICES (ÉTUDE COMPLĖTE D'UN TYPE DONNÉ DE COLLINES}

Le mémoire de Al. Gmatr sera publié dans an prochain numéro de la Iloulle Blanche.

\section{Discussion}

M. Ie Président remercie sincèrement M. Gunat d'aroir présenté un sujet extrèmement austère, sous one forme si facile et si agrable que lon pourrat ne pas se rendre comple des anneses de fravail que represente sa conférence, que kiploxa, s’il vivait encore, aupait probablement appelee un simple conte des collines on.

N. le Président sugferce aux Nembres du Comité Technique de temoigner leur gratilude à M. Gminar ret hes collahorateurs d'E.D.F. en féposant leur avis autorise sur le registre de lenquête publique ouverte dans la région de Saint-Malo-Dinan, e'l vue de la demande de concession de l'usinc de la hance. 
M. Hakgeles, tout en comprenant toute limportance des variations de rendement des turbines en fonction de tels changements de dénivellation, se demande si on n'aurat pas pu étudice cela par des procédés beaucoup plas simples en prenant quelques régimes types de bassiu et en essayant chaque turbine sur ce regime de vidange.

M. Gmatre repond que cela reviendmil à faire l'hypothèse que le régime de vidange est le mème quelle que soit la colline présentee. Or, les debits de collines var rient du simple au double el une tranche d'eau de $10 \mathrm{~cm}$ de hauteur, pompée à maréc haute, pent permettre de sagner $13 \mathrm{~m}$ de hauteur de chute à maré hasse. Les experiences mentionnes par M. Habcerses ont éte faites, mais elles ont montra qu'en turbinant au voisinage des rendements maximum on risquat d'utiliser des volumes deau qui seraical beacoup plus utlles en diatres moments.

M. Gabat rappelle, en staidant d'une figure, que l'exploitation donnant te meilleur rendement global de lusine consistera à fonctionner tantôt à rendemenl maximum des turbines (ligne inférieure), tantôt (aus heures de pointe) à debit de puissance maximum (ligne supéricure du triangle); or, les deux lignes correspondent a les debits donbles l'un de lautre dans les cas interessants, et te problème du calcul des rariations consiste à essayer de passer d'un dobit a l'autre.

M. Hazgetex demande ensuite à M. Gmuat comment il a été déterminé à prendré des turbines de fable débil parmi les différents types adoptes.

M. Gmmar répond que dirers Constructenrs consultés ont propesé a F.D.F. des projets de turbines présentant des collires differentes, et dont certaines correspondaient à de faibles débits : comme il est possible d'inshaller le nombre de turbines voulues, chaque réponse de constructeur est caractériséc par une courbe et un nombre.

Répondant à une remarque de M. Aubent, sur l'encombrement des turbines et son influence sur le prix, M. Grbrat indique qu'E.D.F. a étudié dans le minimum de temps, grâce au diagramme établi, toutes les caractéristiques des turbines proposées par les constructeurs el sollicite des variantes von de ramener les offres it des données comparables.

Répondant à une question de M. Xrzeny sur linfluence du prix des kilowalts-heures produits, M. Gunat précise que co facten peut etre introduit dans les calculs de la môme façon que l'indice de réfraction dans les lois de l'optique.

Sur une demande de M. le président, relative au procédé employé pour les calculs, M. Grbat indique que les dessinateurs travallent jusqu'à présent, comme pour l'établissement de tables de tir, sur des feuilles de calcal préalablement mises au point par les Ingénieurs. II. Ginrar ajoute que, d'autre part, des méthodes de calcul mécanique et electrique sur lesquelles il pense pouvoir faire une communication l'année prochaine, sont à l'étude : soit par machines analogiques, dans lesquelles les collines sont représentées par des surfaces électriques, soit par machines arithmetiques ordinaires; dans les deux cas, la limitation de la puissance de lalternateur a cré une diffeulté de mise au point, maintenant résolue. La theoric de ces calculs mecaniques est done acquise, mais leur économie pose encore certaines queslions: dans le moment il parât plus avantageux d'utiliser les machines usuelles existant sur le marché, ru les ecarts des precisions demandées par les divers calculs qui se présentent dans ce projet prototype, mais après l'xecution de l'usine de la Rance, il semble qu'E.D.F. aura intéré à réaliser une machine spéciale.

La séance est levée et la session est close. 


\section{Tournée Glaciologique 1953}

Malgé l'absence regretlé de M. Messines ou Sovnman.

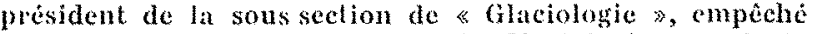
au dernier moment, la "Tourné Glaciologique de la Société Hydrotechnique de France s'est deroulée, cette annee, les 21 ef 23 jullet, sur le Grand Claciet d'Aletseh, par un temps providentiel et avee un plein succes, grâce at concours des Glaciologues el Hydrologues suisses.

Les quarante participants ont visite dans le cadre grandiose du Jungfrajoch (alt. $3.475 \mathrm{~m}$ ) l'Observatoirc Météorologique du Sphinx et l'Institut seientifique, puis parcouru, du Jungfraufrn a la gorge de la Ilassa (all. $1.500 \mathrm{~m}$ ), le plus grand glaeier des Alpes :

Distance horizontale : $22,8 \mathrm{~km}$;

Superficie occupée par le glacier et ses affluents : $146 \mathrm{~km}^{2}$ ¿ la fin du xix siècle, $136 \mathrm{~km}^{2}$ en 1927 ;

Superficie du hassin versant : $205 \mathrm{~km}^{2}$ a la station de Massaboden;

Point culminant du bassin versant: Gross Aletsehhorn $(4.195 \mathrm{~m})$;

Altitude moyenne du bassin versant : $2.920 \mathrm{~m}$;

Limile du névé : $2.950 \mathrm{~m}$;

Rapport les surfaces du névé et du dissipateur: $1,6 / 1$.

An cours de la visite de l'Instilut Scientifique, M. RENaUD rappelle ses recherches sur la cristallographie de la glace (communication à la sous-section de Glaciologie de la S.H.F. du $2 \mathrm{mai} 1950$ ) et la suite qu'il a donnée à cette étude, spécialement en ce qui concerne l'orientation des cristaux de glace en relation avec la texture rubannée et avec la plasticité des glaciers froids.

M. Kasser rappelle ensuite et commente les mesures lopographiques de MM. Haenel, Rogh et Kassen sur le Jungfraufirn et les sondages de $M$. Penutz.

L'ensemble de ces travaux a donné les résultats suivants :

Hauteur de neige: 7 a $0 \mathrm{~m}$ sur les parties supérienres du névé;

Résidu annuel nécessaire pour maintenir l'équilibre du glacier : $4,80 \mathrm{~m}$;

Ablation maximum, mesurée en 1951-52 a l'altitude d'environ $1.700 \mathrm{~m}: 14,90 \mathrm{~m}$;

Vitesse du flacier en surface : 10 à $40 \mathrm{~cm}$ a $1 \mathrm{l}$ Jungfrau; 50 à 60 a la sortie de Konkordia;

Profondeut de la glace dans le névé : $136 \mathrm{~m}$ (forage thermique de Perutz).

Des appareils de mesure du déplacenent et de la viscosité du glacier, en fonctionnement dans la galerie sous-glaciaire des P.T.T., sont présentés par M. KassEx.

On trouvera des renseignements complémentaires dans l'article de MM. Kassen et HaErela : Glaciologische Beobachtungen am Grossen Aletschgletscher (Schweiz. Bauzeitang 70, jy. 1952, $\mathrm{N}^{\circ} 35$ ) dont une traduction est déposée au secrétariat de la S.H.F.

A Konkordia, où les participants ont conché, M. Kassen rappelle l'intérèt special du surcreusement de plus de 200 m du glacier, confirmé par les sondages sismiques de Mothes et Sorge en 1928 et de Suisstrunk et Florin en 1947

Au passage au lac de Märjelen (alt. $2.300 \mathrm{~m}$ ), M. Kassen commente les particularités de ce lac de barrage glaciaire, dont la plus remarquable est celle de pouvoir se vider partiellement ou complètement à des intervalles irréguliers avee une rapidité très variable, soit par débor* dement sur la surface du glacier, soit en empruntant les camaux intraglaciaires atteints par une crevasse on sous l'effet de l'érosion du barrage.

Cette journée s'est terminée par un coup d'œil sur le shlacier de Fiesch et par une conference de M. It hanoine Mantax, professeur et président de la Murithienne, Societe Valaisanne de Sciences Naturelles, preparant la visite a la reserve forestiere d"dlolsell, faite fo lendemain sous sa conduite.

Apres une longue phase préparatoire, 300 ha environ de la foret d'Aletseh, entre 1.700 et $2.200 \mathrm{~m}$ d'allitude, ont éte mis en réserve tolale en 1933. Il est interdit d'cxploiter les bois, de cueillir les plantes, d'y mener lo betail, de chasser.

L'intêtét de eelte forêt d'aroles et de mélezes réside surtout dans le fait de sa position au-dessus du Grand Glacier d'Aletsch. Des coupes exagerées ef le parcours du bélail lavaient affablie; dejà un majeunissement rejouissant se fait sentir. On espère que la foret gagnera lin partie superieure du versant; des terains neufs son mis à découvert par le recul du glacies. On peut y observer la colonisation par des plantes du roisinage. La faune alpine profte aussi largement de la protection dont elle est l'objet.

Des etudes ont été publices sur la ford, les lichens, les mousses, la flore, la fanne, les protozonires du sol, la météorologie.

La beauté de cette région est remarquable tam par la résistance des arbres qui luttent victorieusement contre les conditions hostiles que par les formes du paysage. les glaciers et les grandes sommites. Sur place, $M$. le chanoine Manntran donne de nombreux dètails specialement sur la reproduclion des pins aroles.

A Riederatp, diveres allocutions furent prononcées, par MM. Barrillon, Jost, vice-président, représentant M. Hafrel empeché, Mencanton ancien président de la Commission Helvétique des Glaciers, M. VANN, secrétaire général du Comitatto Glaciologico Ilaliano. Des remerciements furent adressés aux remaruables organisateurs suisses et des toasts furent portés au succes croissant de la Toumee Glaciologique de ha.F., sous l'impulsion de M. Le président Mrsstnes, a la Société Suisse des Seiences Naturelles, au Comilatio Gaciologico Italiano et a la British Glaciological Society.

La Tournee se termina par nue démonstration faite par M. Kassm de l'installation de forage thermique fsystème Caleiali) et de la pose de halises d'ablation dans la zone inferieure du glacier, enfin par la visite de la gorge de la Massa el de la prise d'eau de l'Aletseh Aktienm gesellsehaft à La Lonzat.

On mesure depuis 1922 les debils a la station de jaugeage de Massabodet (all. $687 \mathrm{~m}$ ) a $600 \mathrm{~m}$ de la jonetion arec le Bhone.

Les Suisses admoflent une craporation moyenne de

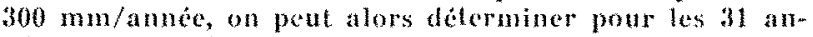
nées de 11920-21 a 1050-51 les valeurs moyenoses suivantes:

Précipitations. . . . . . . . . Diminution des réserves...

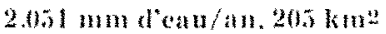
$405 \mathrm{~mm}$ d'eat $/ \mathrm{an} .205 \mathrm{~km} 2$ Hauteur d'écoulement (debit) $2.155 \mathrm{~mm}$ d'ean/an. $205 \mathrm{~km}$ '

Le dessablage de l'usine de la Lonza a causé de sérieuses diffeultés. On les a surmontées à l'äde d'essais sur modèles réduits dans les laboratoires de l'E.P.F. I Zürich. La description générale de l'Aletschwerk, ainsi que de la prise d'eau se trouvent dans les publications suivantes: Schweiz. Banzig., 1950, $n^{\prime \prime} 26$ at 1952, $n^{\prime 2} 10$.

Les participants rejoignent ensuite Blatten, puis Brigut: où a lieu la dislocation.

La Tournée a éte attristée le dernier jour par un accident sans gravité arrivé a l'un des participants. 


\section{Journées de l'Hydraulique}

\section{Alger, 12-14 avril 1954}

La Sochet Hydrolechnique de France organise les Troisiemes Joundes de l'Hydraulique qui se liendront a Alger du 12 an 14 avil 1954.

Surer Étuné: :

PLUIE, BVAPORATION, FLTRATION FT GCOULEMENT

Liste et Titres provisolres

DES COMMUNICATIONS iactobre 1953).

Guestion A

Relations entre les mesures pluviométriques, nivométriques, thermométriques, évaporométriques, et les mesures de débit.

a) Considérations thériques :

L. SEnRA : I. Le controle hydrologique d'un bassin versant. - II. L'influence du relief sur les mesures pluviométriques.

L. Tunc: Le bilan d'eatu des sols: rolations entro les précipitations, l'évaporation et l'écoulement.

L. Kachnolo: Sur une formule de correspondance entre les précipitations atmosphériques et les cotes hydrométriques des cours d'ean.

b) Methodes et appareils de mesure:

G. Mentinger : Difficultés de la mesure des précipitations en montagne.

(i. Letennien: Nivomètre enregistreur basé sur l'absorption d'un rayon radioactif.

G. Rẻménnónas : Quelques exemples de stations de jaugeage à déversoir pour petits cours d'eau.

c) Observations faites sur des bassins versants:

L... Tison : Pluie, évaporation, filtration et écoulement en Belgique.

1. Rower el P. Tovchebeur : Evaporation sur les surfaces d'ea naturelles en Afrique Noire Frangaise.

J. Magrin : Remarques sur quelques relations entre la pluie et les écoulements dans le bassin versant de l'usine de Marèges sur la Dordogne.

C. SAme : Ftude hydrologique de la Haute Medjerdah.

$$
\text { d) Esstis: }
$$

P. Caples : Termin expérimental d'Alrance - études des pertes par éxaporation à l'échelle mensuelle.

J. Zaour : Contributions a l'étude de l'évaporation dans les sables.

\section{Questron $B$}

Relations entre le débit passant dans la partie jaugeable et le débit passant dans le sol au voisinage de la partie jaugeable.

H. Chamayov: Ecoulements souterrains.

1'. Guevel : Comparaison entre les principales méthodes d'étude expérimentale des écoulements souterrains.

M. Mexny, Bonner el Mathiax : Nappe de la plaine alluriale de la rive gauche du Rhône entre Donzère et Mornas.

\section{Questron C}

Considérations statistiques à l'échelle de la décade, du mois, de l'anné; comparaisons entre divers bassins et diverses régions.

L. Habmes : L'hydrologie statistique : nature, objet, méthodes.

(i. Monlat of A. Buhne: Sur Futilisalion judicicuse de liaisons statistiques dans quelques domaines d'hydrologie où interviennent des séries d'observations de lailles differentes.

II. Gansonint ef R. Johansien : Debit des pluies orageuses daus la region parisienne.

M. Pennesset : Précipitations en Tunisie.

A. be Montmann: Intensité et duré des averses enregistrées a Tunis.

1:. Sumavo: Précipitations à Madagascar et aux lles Comores.

J. Emon: L'éraporation au Maroc.

J. Ropien et Mi. Roche : Déficit annuel d'écoulement en pays tropicaux.

M. Pardé: Sur les coefficients d'écoulement des très grandes erues.

A. Coutagne: Etude de quelques correlations hydrométéorologiques régionales.

\section{Question D}

Filtration - Dégravement - Dévasement.

d. Ferrandon : Dynamique des sols perméables: équalions, propagation par ondes.

F. SERre : Consolidation d'une couche d'argile d'épaisseur indéfinie surmontée d'une couche de sable.

$\mathrm{x}$. Michon: Tassement et consolidation des boues de Durance.

G. Schnembli : La mesure in situ de la perméabilité d'un terrain.

L. Escande : Dispositif spécial pour le dégravement d'une prise d'eau.

Ets NeYrPIC : I. Mesure des pertes de charge en milien poreux.

II. Etude des pertes de charge dans les crépines de puits de pompage.

Des visites de laboratoires et d'usines auront lien l'après-midi du mardi 13 avril. Les participants auront

a faire un choix pour ces visites.

Des voyages d'études sont prévus du 19 au 29 avril 1954 en Algérie, Tunisie et Maroc et du $1^{\text {er }}$ au 7 mai en France.

On s'insclit à la Société Hydrotechnique de France, 199 , rue de Grenclle, Paris $(7)$. Tél. INV 13-37 (délai reporté au 31 décembre irrévocablement).

\section{INVITATION AU II CONGRES INTERNATIONAL DES IRRIGATIONS ET DU DRAINAGE}

L*Association Française des Frrigations et du Drainage invite tous les participants des « Journées de lHydraulique » à assister au II $^{\mathrm{C}}$ Congrès International des Irrifrations et du Drainage, çui est organisé à Alger du 15 au 17 avril 1954.

Le programme de ce Congrès est donné dans le Bulletin $n^{*} 2$ de la Commission Internationale des Irrigations et du Drainage.

Pour tous renseignements concernant les activités de l'Association et l'organisation de ce Congrès, s'adresser au :

Secrétariat de l'Association Francaise pour l'Etude des Irigations et du Drainage: Boîte postale 52

Grenoble. 